Supporting Information to Accompany:

\title{
Quantification of the Electrostatic Effect on Redox Potential by Positive Charges in a Catalyst Microenvironment
}

Natalia D. Loewen, ${ }^{\ddagger 1}$ Santanu Pattanayak, ${ }^{\ddagger 1}$ Rolfe Herber, ${ }^{2 *}$ James C. Fettinger, $^{1}$ and Louise A. Berben ${ }^{1 *}$

${ }^{1}$ Department of Chemistry, University of California, Davis, CA 95616

${ }^{2}$ Racah Institute of Physics, The Hebrew University of Jerusalem, Edmond J. Safra campus, Givat Ram, Jerusalem 91904, Israel

$\$$ these authors contributed equally.

email to: laberben@ucdavis.edu

\section{Table of Contents}

\section{Experimental Section}

2. Tables

Table S1. Crystallographic data for Na-7, $\mathrm{Et}_{4} \mathrm{~N}-8$, 9-OTf and 11-OTf 3

Table S2. Selected average interatomic distances $(\AA)$ and selected average angles (deg).

Chart S1. Atom numbering schemes for Table S2.

\section{Figures}

4. Figures S1- S25. ${ }^{1} \mathrm{H},{ }^{13} \mathrm{C},{ }^{31} \mathrm{P}\left\{{ }^{1} \mathrm{H}\right\}$ and ${ }^{19} \mathrm{~F}$ NMR spectra of all the clusters synthesized in this manuscript.

Figure S26. Plot of Fe1-P bond distances against cone angle of phosphines.

Figure S27. Zero field ${ }^{57} \mathrm{Fe}$ Mössbauer spectrum of $\mathrm{Et}_{4} \mathrm{~N}-6$. 
Figure S28. Zero field ${ }^{57} \mathrm{Fe}$ Mössbauer spectrum of Na-1.

Figure S29. IR spectra of clusters synthesized in this manuscript.

Figure S30. IR spectra of $\mathrm{Et}_{4} \mathrm{~N}-1$ in various anhydrous solvents.

Figure S31. Solvatochromic IR study of 9-OTf and 11-OTf . $_{\text {. }}$

Figure S32. DPV spectra of clusters $\mathbf{1}^{-}-\mathbf{1 1}^{3+}$ in acetonitrile under $\mathrm{N}_{2}$.

Figure S33. DPV of $0.05 \mathrm{mM} \mathrm{11-OTf}_{3}$ in acetonitrile in presence of different anion source.

Figure S34. DPV of $0.2 \mathrm{mM} \mathrm{11-OTf}_{3}$ in acetonitrile with increasing ionic strength.

Figure S35. IR spectra of $0.05 \mathrm{mM} \mathrm{11-OTf}_{3}$ in acetonitrile with increasing ionic strength.

\section{References}

\section{Experimental Section}

X-ray structure determination. X-ray diffraction studies for $\mathrm{Na}-7, \mathrm{Et}_{4} \mathrm{~N}-8,8$ 9-I and 11$\mathrm{OTf}_{3}$ were carried out on a Bruker Photon 100 CMOS diffractometer or a Bruker SMART APEXII diffractometer equipped with a CCD detector. ${ }^{1}$ Measurements were carried out at $100 \mathrm{~K}$ or 90 $\mathrm{K}$ using $\mathrm{Mo} \mathrm{K}_{\alpha} 0.71073 \AA$ radiation for $\mathrm{Na}-7, \mathrm{Et}_{4} \mathrm{N-8}, 11-\mathrm{OTf}_{3}$ and at $90 \mathrm{~K}$ using $\mathrm{Cu} \mathrm{K}_{\alpha} 1.54178$ $\AA ̊$ radiation for 9-I. The crystals were mounted on a Kaptan Loop with Paratone-N oil. Initial lattice parameters were obtained from a least-squares analysis of more than 100 centered reflections; these parameters were later refined against all data. Data collected were corrected for Lorentz and polarization effects with Saint ${ }^{2}$ and absorption using Blessing's method and merged as incorporated with the program Sadabs. ${ }^{3}$

Space group assignments were based upon systematic absences, E statistics, and successful refinement of the structures. Structures were solved by direct methods with the aid of successive difference Fourier maps and were refined against all data using the SHELXT and SHELXL-2014 software package. ${ }^{4}$ Thermal parameters for all non-hydrogen atoms were refined anisotropically. Hydrogen atoms, where added, were assigned to ideal positions and refined using a riding model with an isotropic thermal parameter 1.2 times that of the attached carbon atom (1.5 times for methyl hydrogens). For structure $\mathrm{Et}_{4} \mathrm{N-8}$, one of the two nearly identical molecules of interest possessed nearly whole molecule disorder in the core and the associated ligands that was refined to be 0.736:0.264. A variety of restraints were required for structural refinement and those commands included distance restraints (SADI, DFIX), similar thermal motion restraints for atoms that were overlapping or nearly so (SIMU, ISOR). One of the two tetraethylammonium cation was slightly disordered and that refined to 0.93:0.07. Hydrogen atoms were idealized throughout the final refinement. All crystallographic calculations were performed on a Surface Pro5 with Intel i7$7660 \mathrm{U}$ at $2.50 \mathrm{GHz}$ with two cores, four processors and 16GB of extended memory.

Electrochemical measurements. Cyclic voltammograms and differential pulse voltammograms were recorded under a dinitrogen (Praxair, 99.998\%) atmosphere using a $\mathrm{CH}$ Instruments Electrochemical Analyzer Model $1100 \mathrm{C}$ or $620 \mathrm{E}$, a glassy carbon button working electrode $\left(\mathrm{CH}\right.$ Instruments, nominal surface area of $\left.0.0707 \mathrm{~cm}^{2}\right)$, a platinum wire auxiliary electrode and an $\mathrm{Ag} / \mathrm{AgNO}_{3}$ reference electrode with a Vycor tip. Reported potentials are all referenced to the SCE couple. Ferrocene was used as an external standard where $E_{1 / 2}$ 
ferrocene/ferrocenium is $0.4 \mathrm{~V}$ vs. $\mathrm{SCE}$ in acetonitrile. $\mathrm{Bu}_{4} \mathrm{NBF}_{4}$ was prepared according to the literature, 5 and was purified by dissolving in ethyl acetate and washing with milliQ water followed by recrystallization from boiling ethyl acetate layered with n-hexane, and dried under vacuum at $70^{\circ} \mathrm{C}$ for 48 hours before use. Non-aqueous electrolyte solutions were stored over $3 \AA$ molecular sieves which had been activated by heating under vacuum at $200{ }^{\circ} \mathrm{C}$ for at least 72 hours. For differential pulse voltammetry (DPV) the parameters are: Incr E $=0.04 \mathrm{~V}$, Amplitude $=0.05 \mathrm{~V}$, Pulse width $=0.05 \mathrm{~s}$, Sample width $=0.0167 \mathrm{~s}$, Pulse period $=0.5 \mathrm{~s}$.

Other Physical Measurements. ${ }^{1} \mathrm{H}-\mathrm{NMR},{ }^{13} \mathrm{C}-\mathrm{NMR},{ }^{19} \mathrm{~F}-\mathrm{NMR}$ and ${ }^{31} \mathrm{P}-\mathrm{NMR}$ spectra were recorded at ambient temperature using a Varian 300 or $600 \mathrm{MHz}$ spectrometer, a Bruker 400 $\mathrm{MHz}$ TopSpin spectrometer, or a Bruker $800 \mathrm{MHz}$ TopSpin spectrometer. Chemical shifts were referenced to the residual solvent peaks. ${ }^{31} \mathrm{P}-\mathrm{NMR}$ spectra were referenced using an external $\mathrm{H}_{3} \mathrm{PO}_{4}$ standard (chemical shift of $\mathrm{H}_{3} \mathrm{PO}_{4}=0 \mathrm{ppm}$ ). ${ }^{19} \mathrm{~F}-\mathrm{NMR}$ spectra were referenced using an external standard (chemical shift of $\mathrm{CF}_{3} \mathrm{COOH}=-76.55 \mathrm{ppm}$ ). Infra-red spectra were recorded in a sealed liquid cell (SPECAC) on a Bruker Alpha Infra-red spectrometer.

Ruling out Ion Pairing Effects. It is possible that clusters with varied charges are subject to ion pairing effects that influence observed redox potentials or $v_{\mathrm{CO}}$ bands. ${ }^{6}$ To study this, we collected DPV's for $\mathbf{1 1}^{3+}$ in $0.1 \mathrm{M} \mathrm{Bu}_{4} \mathrm{NBF}_{4} \mathrm{MeCN}$ solution with 100 equivalents of various anions, $\mathrm{Bu}_{4} \mathrm{NPF}_{6}, \mathrm{Me}_{4} \mathrm{NBF}_{4}$ or $\mathrm{NaBAr}_{4}$, added to the solution and no change in the electrochemical data was observed (Figure S32). DPV's collected in $\mathrm{MeCN}$ with $\left[\mathrm{Bu}_{4} \mathrm{NBF}_{4}\right]$ spanning $0.1-0.5 \mathrm{M}$ also showed no change in $E_{\mathrm{p}}$ (Figure S33). We collected IR spectra for $\mathbf{1 1}^{3+}$ in solutions containing $\left[\mathrm{Bu}_{4} \mathrm{NBF}_{4}\right]$ spanning $0.1-0.5 \mathrm{M}$, and those showed no variance in the band shape or the full width half maximum (fwhm) values of the $v_{\mathrm{CO}}$ bands (Table 1, Figure S34). ${ }^{7}$ Taken together, this suggests that ion-pairing does not significantly influence electrochemical or IR spectroscopic data.

Preparation of Compounds. All manipulations were carried out using standard Schlenk or glove-box techniques under a dinitrogen atmosphere. Unless otherwise noted, solvents were deoxygenated and dried by thorough sparging with Argon (Praxair, 99.998\%) gas followed by passage through an activated alumina column. Deuterated solvents were purchased from Cambridge Isotopes Laboratories, Inc., and were degassed before use. Compounds $\left[\mathrm{Et}_{4} \mathrm{~N}\right]\left[\mathrm{Fe}_{4} \mathrm{~N}(\mathrm{CO})_{12}\right]\left(\mathrm{Et}_{4} \mathrm{~N}-\mathbf{1}\right){ }_{,}^{8}\left[\mathrm{Et}_{4} \mathrm{~N}\right]\left[\mathrm{Fe}_{4} \mathrm{~N}(\mathrm{CO})_{11}\left(\mathrm{PPh}_{2} \mathrm{pyy}\right)\right]\left(\mathrm{Et}_{4} \mathrm{~N}-5\right),{ }^{9}$ $\left[\mathrm{Et}_{4} \mathrm{~N}\right]\left[\mathrm{Fe}_{4} \mathrm{~N}(\mathrm{CO})_{11}\left(\mathrm{PPh}_{3}\right)\right]\left(\mathrm{Et}_{4} \mathrm{~N}-6\right),{ }^{10}$ 1, 3, 5-azido-7-phosphadamantane (PTA), and 1- $\mathrm{N}$ methyl-3, 5-azido-7-phosphadamantanium triflate ([MePTA]OTf) ${ }^{11}$ were prepared following previously reported methods. All other reagents were purchased from commercial vendors and used without further purification.

$\left[\mathbf{E t}_{4} \mathbf{N}\right]\left[\mathbf{F e}_{4} \mathbf{N}(\mathbf{C O})_{11} \mathbf{P}(\mathbf{O P h})_{3}\right]\left(\mathbf{E t}_{\mathbf{4}} \mathbf{N}-2\right)$. [Na(diglyme $\left.)_{2}\right]\left[\mathrm{Fe}_{4} \mathrm{~N}(\mathrm{CO})_{12}\right](107 \mathrm{mg}, 0.124$ mmol and 1 eq. of $\mathrm{P}(\mathrm{OPh})_{3}(38.39 \mathrm{mg}, 0.124 \mathrm{mmol})$ were dissolved in $3 \mathrm{~mL}$ THF in a vial sealed with a Teflon lined screw cap. The reaction mixture was heated at $60{ }^{\circ} \mathrm{C}$ for $16 \mathrm{~h}$. After it was cooled, the reaction mixture was filtered over a $2 \mathrm{~cm}$ Celite plug, and an equal volume of hexane was added so that a black precipitate formed. The black powder $(96.4 \mathrm{mg}, 67.9 \%)$ was collected by filtration and then used in a reaction with $\mathrm{Et} 4 \mathrm{NCl}$ (15.3mg, $0092 \mathrm{mmol})$ in $\mathrm{CH}_{2} \mathrm{Cl}_{2}$. A black crystalline powder $\left(72.6 \mathrm{mg}, 88 \%\right.$ yield) was obtained from a concentrated $\mathrm{Et}_{2} \mathrm{O}$ solution held at $-16^{\circ} \mathrm{C}$ for $2-3$ days. ${ }^{1} \mathrm{H}$ NMR $\left(599 \mathrm{MHz}, \mathrm{CDCl}_{3}-d_{1}\right) \delta 7.59-6.72(\mathrm{~m}, 15 \mathrm{H}, \mathrm{Ar})$, $2.98\left(\mathrm{~s}, 8 \mathrm{H}, \mathrm{NCH}_{2} \mathrm{CH}_{3}\right), 1.45-0.87\left(\mathrm{~m}, 12 \mathrm{H}, \mathrm{NCH}_{2} \mathrm{CH}_{3}\right) .{ }^{13} \mathrm{C} \mathrm{NMR}\left(101 \mathrm{MHz}, \mathrm{CD}_{3} \mathrm{CN}\right) \delta$ 
217.72 (s, CO), 216.87 (s, CO), $216.73(\mathrm{~s}, \mathrm{CO}), 215.00(\mathrm{~s}, \mathrm{CO}), 152.39$ (d, $\left.J_{\mathrm{PC}}=8.6 \mathrm{~Hz}, \mathrm{Ar}\right)$, 130.55 (s, Ar), 125.72 (s, Ar), 125.25 (s, Ar), 122.23 (d, $J_{\mathrm{PC}}=4.4 \mathrm{~Hz}, \mathrm{Ar}$ ), 68.14 (s, THF), 52.95 (s, $\mathrm{NCH}_{2} \mathrm{CH}_{3}$ ), 26.13 (s, THF), $7.54\left(\mathrm{~s}, \mathrm{NCH}_{2} \mathrm{CH}_{3}\right) .{ }^{31} \mathrm{P}\left\{{ }^{1} \mathrm{H}\right\} \mathrm{NMR}\left(162 \mathrm{MHz}, \mathrm{C}_{6} \mathrm{D}_{6}\right) \delta 165.39$ ppm. IR $v_{\mathrm{CO}}(\mathrm{MeCN}): 2045$ (w), 2018 (vw), 1996 (s), 1977 (vs), 1970 (sh) cm ${ }^{-1}$. Combustion analysis calculated for $\mathrm{C}_{37} \mathrm{H}_{35} \mathrm{Fe}_{4} \mathrm{~N}_{2} \mathrm{O}_{14} \mathrm{P}: \mathrm{C}, 45.07 ; \mathrm{H}, 3.58 ; \mathrm{N}, 2.84$. Found: $\mathrm{C}, 45.03 ; \mathrm{H}, 3.66$; N, 2.73.

$\left[\mathbf{E t}_{\mathbf{4}} \mathbf{N}\right]\left[\mathbf{F e}_{\mathbf{4}} \mathbf{N}(\mathbf{C O})_{11} \mathbf{P}(\mathbf{4}-\mathbf{F P h})_{3}\right]\left(\mathbf{E t}_{\mathbf{4}} \mathbf{N}-3\right)$. $\left[\mathrm{Na}(\text { diglyme })_{2}\right]\left[\mathrm{Fe}_{4} \mathrm{~N}(\mathrm{CO})_{12}\right](148 \mathrm{mg}, 0.171$ $\mathrm{mmol})$ and 1 eq. of $\mathrm{P}(4-\mathrm{FPh})_{3}(54.12 \mathrm{mg}, 0.171 \mathrm{mmol})$ were dissolved in $3 \mathrm{~mL}$ THF in a vial sealed with a Teflon lined screw cap. The reaction mixture was heated at $60{ }^{\circ} \mathrm{C}$ for 12 hours and then filtered through a $2 \mathrm{~cm}$ Celite plug. The resulting solid was washed with hexanes to afford $184.7 \mathrm{mg}(93.6 \%, 0.16 \mathrm{mmol})$ black powder which is a $\mathrm{Na}$ salt of 3- Reaction of the black powder with $\mathrm{Et}_{4} \mathrm{NCl}$ (29.2 $\mathrm{mg}, 0.0176 \mathrm{mmol}$ ) in $\mathrm{CH}_{2} \mathrm{Cl}_{2}$ was performed over $2 \mathrm{~h}$, and the reaction solution was filtered through a small Celite plug. The $\mathrm{CH}_{2} \mathrm{Cl}_{2}$ was removed under vacuum and the resulting black powder was washed three times with $\mathrm{Et}_{2} \mathrm{O}(3 \times 4 \mathrm{~mL})$ and dried in vacuo to afford $\mathrm{Et}_{4} \mathrm{~N}-3$ (107 mg, $67 \%$ yield). ${ }^{1} \mathrm{H}$ NMR $\left(400 \mathrm{MHz}, \mathrm{CDCl}_{3}-d_{1}\right) \delta 7.57$ (q, $J=$ 7.6, 7.1 Hz, 6H, Ar), 7.10 (t, $J=8.4 \mathrm{~Hz}, 6 \mathrm{H}, \mathrm{Ar}), 3.15$ (q, $J=7.3 \mathrm{~Hz}, 8 \mathrm{H}, \mathrm{NCH}_{2} \mathrm{CH}_{3}$ ), 1.30 (t, $J$ $\left.=7.6 \mathrm{~Hz}, 12 \mathrm{H}, \mathrm{NCH}_{2} \mathrm{CH}_{3}\right) .{ }^{13} \mathrm{C} \mathrm{NMR}\left(101 \mathrm{MHz}, \mathrm{CDCl}_{3}-d_{1}\right) \delta 216.44(\mathrm{~s}, \mathrm{CO}), 213.15(\mathrm{~s}, \mathrm{CO})$, $164.00\left(\mathrm{~d}, J_{\mathrm{FC}}=251.7 \mathrm{~Hz}, \mathrm{Ar}\right), 136.02(\mathrm{~d}, J=12.1,8.2 \mathrm{~Hz}, \mathrm{Ar}) 130.72$ (d, $\left.J=39.8 \mathrm{~Hz}, \mathrm{Ar}\right)$, $115.70(\mathrm{dd}, J=21.1,9.8 \mathrm{~Hz}, \mathrm{Ar}), 52.88\left(\mathrm{~s}, \mathrm{NCH}_{2} \mathrm{CH}_{3}\right), 7.71\left(\mathrm{~s}, \mathrm{NCH}_{2} \mathrm{CH}_{3}\right) .{ }^{31} \mathrm{P}\left\{{ }^{1} \mathrm{H}\right\} \mathrm{NMR}(162$ $\left.\mathrm{MHz}, \mathrm{CDCl}_{3}-d_{1}\right) \delta 65.23 \mathrm{ppm} .{ }^{19} \mathrm{~F}\left\{{ }^{1} \mathrm{H}\right\} \mathrm{NMR}\left(376 \mathrm{MHz}, \mathrm{CDCl}_{3}-d_{1}\right) \delta-110.25 \mathrm{ppm}$. IR $v_{\mathrm{CO}}(\mathrm{MeCN}): 2037$ (m), 2018 (w) 1988 (vs), 1974 (s), 1975 (sh), 1931 (vw) cm-1. Combustion analysis calculated for $\mathrm{C}_{37} \mathrm{H}_{32} \mathrm{~F}_{3} \mathrm{Fe}_{4} \mathrm{~N}_{2} \mathrm{O}_{11} \mathrm{P}: \mathrm{C}, 44.80 ; \mathrm{H}, 3.25 ; \mathrm{N}, 2.82$. Found: $\mathrm{C}, 44.23 ; \mathrm{H}, 3.52$; $\mathrm{N}, 2.83$.

$\left(\mathbf{E t}_{\mathbf{4}} \mathbf{N}\right)\left[\mathbf{F e}_{\mathbf{4}} \mathbf{N}(\mathbf{C O})_{1 \mathbf{1 1}}\left(\mathbf{P E t}_{\mathbf{3}}\right)\right]\left(\mathbf{E t}_{\mathbf{4}} \mathbf{N}-\mathbf{4}\right)$. [Na(diglyme $\left.)_{2}\right]\left[\mathrm{Fe}_{4} \mathrm{~N}(\mathrm{CO})_{12}\right](50 \mathrm{mg}, 0.058 \mathrm{mmol})$ and $\mathrm{PEt}_{3}(6.83 \mathrm{mg}, 0.058 \mathrm{mmol})$ were dissolved in $3 \mathrm{~mL}$ THF in a vial sealed with a Teflon lined screw cap. The reaction mixture was heated at $60^{\circ} \mathrm{C}$ for 16 hours. After cooling, the reaction mixture was filtered through a $2 \mathrm{~cm}$ Celite plug and then the solvent was removed in vacuo to afford $46.28 \mathrm{mg}$ of the impure product. A solution of the unpurified $\mathrm{Na}$ salt of $4^{-}$and $\mathrm{Et}_{4} \mathrm{NCl}$ $(8.83 \mathrm{mg}, 0.053 \mathrm{mmol})$ was stirred in dichloromethane for $2 \mathrm{~h}$. After the reaction mixture was filtered through celite, the solvent was removed in vacuo, and the product was recrystallized from $2 \mathrm{~mL}$ of an 8:2 $\mathrm{Et}_{2} \mathrm{O}$-THF solution held at room temperature. After 2 - $3{\text { days, } \mathrm{Et}_{4} \mathrm{~N}-4 \text { was }}$ isolated as a black crystalline powder $(36.39 \mathrm{mg}, 95 \%$ yield $) .{ }^{1} \mathrm{H} \mathrm{NMR}\left(599 \mathrm{MHz}, \mathrm{CDCl}_{3}-d_{1}\right) \delta$ $3.15\left(\mathrm{q}, J_{\mathrm{HH}}=7.1 \mathrm{~Hz}, 8 \mathrm{H}, \mathrm{NCH}_{2} \mathrm{CH}_{3}\right), 1.75\left(\mathrm{p}, J=7.7 \mathrm{~Hz}, 6 \mathrm{H}, \mathrm{PCH}_{2} \mathrm{CH}_{3}\right), 1.30\left(\mathrm{t}, J_{\mathrm{HH}}=7.3 \mathrm{~Hz}\right.$, $\left.12 \mathrm{H}, \mathrm{NCH}_{2} \mathrm{CH}_{3}\right), 1.24\left(\mathrm{dt}, J_{\mathrm{PH}}=15.3,7.5 \mathrm{~Hz}, 9 \mathrm{H}, \mathrm{PCH}_{2} \mathrm{CH}_{3}\right) \cdot{ }^{13} \mathrm{C} \mathrm{NMR}\left(101 \mathrm{MHz}, \mathrm{CDCl}_{3}\right) \delta$ 219.09 (s, CO), 218.55 (s, CO), 215.52 (s, CO), $52.74\left(\mathrm{~s}, \mathrm{NCH}_{2} \mathrm{CH}_{3}\right), 19.97$ (d, $J_{\mathrm{PC}}=24.2 \mathrm{~Hz}$, $\left.\mathrm{PCH}_{2} \mathrm{CH}_{3}\right), 8.12\left(\mathrm{~s}, \mathrm{NCH}_{2} \mathrm{CH}_{3}\right), 7.72\left(\mathrm{~s}, \mathrm{PCH}_{2} \mathrm{CH}_{3}\right) .{ }^{31} \mathrm{P}\left\{{ }^{1} \mathrm{H}\right\} \mathrm{NMR}\left(400 \mathrm{MHz}, \mathrm{CD}_{3} \mathrm{CN}\right): 58.34$ ppm. IR $v_{\mathrm{CO}}(\mathrm{MeCN}): 2035$ (m), 2019 (vw), 1981 (vs), 1966 (s), 1960 (sh), 1928 (w) cm-1. Combustion analysis calculated for $\mathrm{C}_{25} \mathrm{H}_{35} \mathrm{Fe}_{4} \mathrm{~N}_{2} \mathrm{O}_{11} \mathrm{P} \cdot 0.8$ (THF): C, 39.77; H, 4.90; N, 3.29. Found: C, 39.69; H, 4.95; N, 3.21.

$\left(\mathbf{E t}_{\mathbf{4}} \mathbf{N}\right)\left[\mathbf{F e}_{\mathbf{4}} \mathbf{N}(\mathbf{C O})_{\mathbf{1 0}}(\mathbf{P T A})_{\mathbf{2}}\right]\left(\mathbf{E t}_{\mathbf{4}} \mathbf{N}-\mathbf{7}\right)$. $\left[\mathrm{Na}(\text { diglyme })_{2}\right]\left[\mathrm{Fe}_{4} \mathrm{~N}(\mathrm{CO})_{12}\right](105 \mathrm{mg}, 0.12$ mmols) and PTA (39 mg, $0.24 \mathrm{mmol}$ ) were added to a vial with $3 \mathrm{ml}$ THF. The reaction mixture was sealed with a Teflon-lined screw cap and heated at $60^{\circ} \mathrm{C}$ for 2 hours before the reaction was 
cooled. The solution was then filtered through a $2 \mathrm{~cm}$ celite plug and the THF was removed in vacuo to afford $120 \mathrm{mg}(0.11 \mathrm{mmol})$ of Na-7 (90 \% yield). Diffraction-quality crystals were obtained from a concentrated THF solution of Na-7 layered with hexane. ${ }^{1} \mathrm{H}$ NMR $(400 \mathrm{MHz}$, $\mathrm{CD}_{3} \mathrm{CN}$ ): 3.30 (br s, 12H), 3.47 (br s, $\left.8 \mathrm{H}\right), 3.54$ (br s, 8H), $4.05(\mathrm{~s}, 12 \mathrm{H}), 4.42(\mathrm{~s}, 12 \mathrm{H}) .{ }^{13} \mathrm{C} \mathrm{NMR}$ $\left(101 \mathrm{MHz}, \mathrm{CD}_{3} \mathrm{CN}\right): 58.84\left(\mathrm{~s}, \mathrm{OCH}_{3}\right), 70.61\left(\mathrm{~s}, \mathrm{OCH}_{2}\right), 72.30\left(\mathrm{~s}, \mathrm{OCH}_{2}\right), 56.19\left(\mathrm{~d}, J_{\mathrm{P}, \mathrm{C}}=14.0\right.$ $\mathrm{Hz}), 73.38\left(\mathrm{~d}, J_{\mathrm{P}, \mathrm{C}}=7.0 \mathrm{~Hz}\right), 219.31(\mathrm{~s}, \mathrm{CO}), 219.34(\mathrm{~s}, \mathrm{CO}), 220.66$ (s, CO), 225.15 (s, CO). ${ }^{31} \mathrm{P}\left\{{ }^{1} \mathrm{H}\right\}$ NMR (162 MHz, THF): -13.68 ppm. IR $v_{\mathrm{CO}}$ (THF): 2006 (m), 1959 (vs), 1937 (vs), 1910 (m) $\mathrm{cm}^{-1}$. Combustion analysis calculated for $\mathrm{C}_{42} \mathrm{H}_{69} \mathrm{Fe}_{4} \mathrm{~N}_{8} \mathrm{Na}_{2} \mathrm{O}_{19} \mathrm{P}_{2} \cdot\left(\mathrm{CH}_{3} \mathrm{CN}\right): \mathrm{C}, 36.13 ; \mathrm{H}$, 4.41; N, 10.21. Found: $\mathrm{C}, 35.97 ; \mathrm{H}, 4.50 ; \mathrm{N}, 10.68$. The $\mathrm{Et}_{4} \mathrm{~N}$ salt of $7^{-}$was also prepared. Reaction of $50 \mathrm{mg}(0.044 \mathrm{mmol}) \mathrm{Na}-7$ with $\mathrm{Et}_{4} \mathrm{NCl}\left(6 \mathrm{mg}, 1\right.$ equiv.) in $1 \mathrm{ml} \mathrm{CH}_{2} \mathrm{Cl}_{2}$ was performed over $2 \mathrm{~h}$, and then the reaction solution was filtered through a small Celite plug. The dichloromethane was removed under vacuum and the resulting black powder was washed three times with $\mathrm{Et}_{2} \mathrm{O}(3 \times 2 \mathrm{~mL})$ and dried in vacuo to afford $\mathrm{Et}_{4} \mathrm{~N}-7\left(32 \mathrm{mg}, 77 \%\right.$ yield). The ${ }^{1} \mathrm{H}$, ${ }^{31} \mathrm{P}\left\{{ }^{1} \mathrm{H}\right\}$ NMR, and IR spectra, and the DPV electrochemical data for $\mathrm{Et}_{4} \mathrm{~N}-7$ were consistent with those recorded for Na-7.

$\left(\mathbf{E t}_{4} \mathbf{N}\right)\left[\mathbf{F e}_{4} \mathbf{N}(\mathbf{C O})_{10}\left(\mathbf{P E t}_{3}\right)_{2}\right]\left(\mathbf{E t}_{4} \mathbf{N - 8}\right)$. [Na(diglyme $\left.)_{2}\right]\left[\mathrm{Fe}_{4} \mathrm{~N}(\mathrm{CO})_{12}\right](217 \mathrm{mg}, 0.25 \mathrm{mmol})$ and $\mathrm{PEt}_{3}(74 \mu \mathrm{L}, 0.5 \mathrm{mmol})$ were dissolved in $5 \mathrm{~mL}$ THF in a vial sealed with a Teflon lined screw cap. The reaction mixture was heated at $60^{\circ} \mathrm{C}$ for $16 \mathrm{~h}$. After cooling, the reaction mixture was filtered through a $2 \mathrm{~cm}$ Celite plug. Hexanes $(4 \mathrm{~mL})$ was added to the filtrate and a black oil was obtained and dried in vacuo. A solution of the impure product and $\mathrm{Et}_{4} \mathrm{NCl}(41.39 \mathrm{mg}, 0.25$ $\mathrm{mmol}$ ) were stirred in dichloromethane for $2 \mathrm{~h}$ and then the reaction solution was filtered through celite before the solvent was removed in vacuo. X-ray quality crystals of $\mathrm{Et}_{4} \mathrm{~N}-8(185.87 \mathrm{mg}$, $93 \%$ yield) were grown from concentrated $\mathrm{Et}_{2} \mathrm{O}$ solution held at room temperature for 3 days. ${ }^{1} \mathrm{H}$ NMR (400 MHz, $\left.\mathrm{CDCl}_{3}-d_{1}\right) \delta 3.10$ (q, $\left.J=6.9 \mathrm{~Hz}, 8 \mathrm{H}, \mathrm{NCH}_{2}\right), 1.83$ (p, $J=7.7 \mathrm{~Hz}, 12 \mathrm{H}, \mathrm{PCH}_{2}$ ), 1.25 (overlapping dt and t, $\left.30 \mathrm{H}, 12 \mathrm{H}, \mathrm{NCH}_{2} \mathrm{CH}_{3} ; 18 \mathrm{H}, \mathrm{PCH}_{2} \mathrm{CH}_{3}\right) .{ }^{13} \mathrm{C} \mathrm{NMR}(400 \mathrm{MHz}$, Chloroform-d): $\delta 8.76\left(\mathrm{~d}, J_{\mathrm{PC}} 1.9 \mathrm{~Hz}, \mathrm{PCH}_{2} \mathrm{CH}_{3}\right), 8.86\left(\mathrm{NCH}_{2} \mathrm{CH}_{3}\right), 21.41\left(\mathrm{~d}, J_{\mathrm{PC}} 23.9 \mathrm{~Hz}, \mathrm{PCH}_{2}\right)$, $53.34\left(\mathrm{NCH}_{2}\right), 213.75$ (s, $\left.\mathrm{CO}\right), 221.71$ (s, $\left.\mathrm{CO}\right) .{ }^{31} \mathrm{P}\left\{{ }^{1} \mathrm{H}\right\} \mathrm{NMR}\left(400 \mathrm{MHz}, \mathrm{CD}_{3} \mathrm{CN}\right): 50.66 \mathrm{ppm}$. IR $\left(v_{\mathrm{CO}}\right)(\mathrm{MeCN}): 2000(\mathrm{~m}), 1951(\mathrm{vs}), 1930(\mathrm{~s}), 1904(\mathrm{w})$. Combustion analysis calculated for $\mathrm{C}_{30} \mathrm{H}_{50} \mathrm{Fe}_{4} \mathrm{~N}_{2} \mathrm{O}_{10} \mathrm{P}_{2}$ : C, 40.76; H, 5.70; N, 3.17. Found: 40.98; H, 5.88; N, 3.22.

$\left[\mathbf{F e}_{4} \mathbf{N}(\mathbf{C O})_{10}\right.$ ('MePTA) $\left.{ }_{2}\right]$ OTf (9-OTf). [Na(diglyme $\left.)_{2}\right]\left[\mathrm{Fe}_{4} \mathrm{~N}(\mathrm{CO})_{12}\right](120 \mathrm{mg}, 0.14 \mathrm{mmol})$ and [MePTA]OTf ( $89 \mathrm{mg}, 0.28 \mathrm{mmol}$ ) were dissolved in $3 \mathrm{~mL}$ THF in a vial sealed with a Teflon lined screw cap. The reaction mixture was heated at $60^{\circ} \mathrm{C}$ for $2 \mathrm{~h}$ before cooling. The reaction mixture was filtered through a $2 \mathrm{~cm}$ celite plug, and then the solvent was removed in vacuo. The remaining dark solids were stirred in $10 \mathrm{~mL}$ dichloromethane for one hour, the solids were allowed to settle, and the dichloromethane was removed using a Pasteur pipette. The remaining solids were extracted into $5 \mathrm{~mL}$ THF and $10 \mathrm{~mL}$ of $\mathrm{Et}_{2} \mathrm{O}$ was added dropwise with stirring. The resulting mixture was filtered to afford 9-OTf ( $75 \mathrm{mg}, 53 \%$ yield). ${ }^{1} \mathrm{H}$ NMR (400 MHz, $\left.\mathrm{CD}_{3} \mathrm{CN}\right): 2.76(\mathrm{~s}, 6 \mathrm{H}), 3.93-4.11(\mathrm{~m}, 8 \mathrm{H}), 4.32(\mathrm{br} \mathrm{s}, 4 \mathrm{H}), 4.34-4.45(\mathrm{~m}, 4 \mathrm{H}), 4.78-4.92(\mathrm{~m}, 8 \mathrm{H})$. ${ }^{13} \mathrm{C}$ NMR $\left(101 \mathrm{MHz}, \mathrm{CD}_{3} \mathrm{CN}\right): 49.96\left(\mathrm{~s}, \mathrm{NCH}_{3}\right), 52.28\left(\mathrm{~d}, J_{\mathrm{P}, \mathrm{C}}=14.1 \mathrm{~Hz}\right), 60.86\left(\mathrm{~d}, J_{\mathrm{P}, \mathrm{C}}=6.6\right.$ $\mathrm{Hz}), 69.91\left(\mathrm{~d}, J_{\mathrm{P}, \mathrm{C}}=6.4 \mathrm{~Hz}\right), 81.41(\mathrm{~s}), 217.38(\mathrm{~s}, \mathrm{CO}), 217.47(\mathrm{~s}, \mathrm{CO}), 217.95(\mathrm{~s}, \mathrm{CO}), 219.08$ (s, CO). ${ }^{31} \mathrm{P}\left\{{ }^{1} \mathrm{H}\right\}$ NMR (162 MHz, $\mathrm{CD}_{3} \mathrm{CN}$ ): $2.18 \mathrm{ppm}$. IR $v_{\mathrm{CO}}$ (THF): 2017 (m), 1972 (vs), 1949 (vs), 1921 (s) $\mathrm{cm}^{-1}$. Combustion analysis calculated for $\mathrm{C}_{25} \mathrm{H}_{30} \mathrm{~F}_{3} \mathrm{Fe}_{4} \mathrm{~N}_{7} \mathrm{O}_{13} \mathrm{P} 2 \mathrm{~S} \cdot 0.7$ (THF) -2( $\left.\mathrm{H}_{2} \mathrm{O}\right): \mathrm{C}, 30.43 ; \mathrm{H}, 3.64 ; \mathrm{N}, 8.93$. Found: $\mathrm{C}, 30.70 ; \mathrm{H}, 3.70 ; \mathrm{N}, 8.63$. 9-I was prepared using 
the same procedure from $\left[\mathrm{Na}(\text { diglyme })_{2}\right]\left[\mathrm{Fe}_{4} \mathrm{~N}(\mathrm{CO})_{12}\right]$ and [MePTA]I. Crystals suitable for X-ray diffraction were grown from a THF/toluene solution of 9-I stored at $-15{ }^{\circ} \mathrm{C}$ for one week.

$\left.\mathbf{K}_{\mathbf{F}} \mathbf{F e}_{\mathbf{4}} \mathbf{N}(\mathbf{C O})_{\mathbf{8}}\left(\mathbf{P E t}_{\mathbf{3}}\right)_{\mathbf{4}}\right] \mathbf{( \mathbf { K } - 1 0 )}$. THF $(20 \mathrm{~mL})$ and $511 \mu \mathrm{L}(3.47 \mathrm{mmol}) \mathrm{Et}_{3} \mathrm{P}$ were added to $500 \mathrm{mg}(0.578 \mathrm{mmol})$ of $\left[\mathrm{Na}(\text { diglyme })_{2}\right]\left[\mathrm{Fe}_{4} \mathrm{~N}(\mathrm{CO})_{12}\right]$ in a $50 \mathrm{~mL}$ oven dried Schlenk flask. The resulting solution was heated at reflux for $2 \mathrm{~h}$ before benzoic acid $(84.72 \mathrm{mg}, 0.693 \mathrm{mmol})$ was added. The THF solution was heated at reflux for another $24 \mathrm{~h}$ before the solvent was removed under vacuum. The resulting deep brown precipitate was washed twice with $10 \mathrm{~mL}$ water to remove any salts before the product was dissolved in hexane $(10 \mathrm{~mL})$, filtered through Celite. The hexanes solution was held at $-15^{\circ} \mathrm{C}$ for two days and then brown crystals of $\mathrm{H}-\mathbf{1 0}$ collected (192 $\mathrm{mg}, 47 \%)$ and used without further purification. $\mathrm{HFe}_{4} \mathrm{~N}(\mathrm{CO})_{8}\left(\mathrm{PEt}_{3}\right)_{4}(110 \mathrm{mg}, 0.117 \mathrm{mmol})$ and $\mathrm{KO}^{\mathrm{t} B u}(13.3 \mathrm{mg}, 0.119 \mathrm{mmol})$ were combined in a $20 \mathrm{~mL}$ vial and then $5 \mathrm{ml}$ of dry THF was added. The vial was sealed with a Teflon lined screw cap and heated at $60{ }^{\circ} \mathrm{C}$ for $6 \mathrm{~h}$. After colling to room temperature, the reaction mixture was filtered through glass microfiber filter paper, THF was removed in vacuo and the solid was washed with $\mathrm{Et}_{2} \mathrm{O}(2 \times 5 \mathrm{~mL})$. The resulting black powder was dried under vacuum to afford $\mathrm{K}-10$ (73.4 mg, $71 \%$ yield). ${ }^{1} \mathrm{H}$ NMR $\left(400 \mathrm{MHz}, \mathrm{C}_{6} \mathrm{D}_{6}\right): 2.8-.57$ (m, 24H), 1.49-0.03 (m, 36H); ${ }^{31} \mathrm{P}\left\{{ }^{1} \mathrm{H}\right\}$ NMR $\left(400 \mathrm{MHz}, \mathrm{C}_{6} \mathrm{D}_{6}\right): 42.82,42.19 ;{ }^{13} \mathrm{C}\left\{{ }^{1} \mathrm{H}\right\} \mathrm{NMR}(800$ $\left.\mathrm{MHz}, \mathrm{C}_{6} \mathrm{D}_{6}\right): \delta 224.73$ (s, CO), $223.11(\mathrm{~S}, \mathrm{CO}), 220.34$ (d, J=11.5 Hz, CO), 215.46 (d, J=23.5 $\mathrm{Hz}, \mathrm{CO}$ ), 20.50 (d, $J_{\mathrm{PC}}=21.8 \mathrm{~Hz}, \mathrm{PCH}_{2} \mathrm{CH}_{3}$ ), 8.29 (dd, $J_{\mathrm{PC}}=42.0,3.5 \mathrm{~Hz}, \mathrm{PCH}_{2} \mathrm{CH}_{3}$ ). IR (MeCN): $v_{\mathrm{CO}} 1968(\mathrm{vw}), 1952(\mathrm{w}), 1912(\mathrm{vs}), 1902(\mathrm{~s}), 1862(\mathrm{~m})$. Combustion analysis calculated for $\mathrm{C}_{32} \mathrm{H}_{60} \mathrm{Fe}_{4} \mathrm{KNO}_{8} \mathrm{P}_{4} \bullet$ (THF): C, 41.37; H, 6.56; N, 1.34. Found: C, 41.33; H, 6.64; N, 1.35 .

$\left.\left[\mathrm{Fe}_{\mathbf{4}} \mathbf{N}(\mathbf{C O})_{\mathbf{8}} \text { ( }^{\text {MePTA }}\right)_{4}\right]\left(\right.$ OTf $_{3}\left(\mathbf{( 1 1 - O T f}_{3}\right) \cdot$ A $100 \mathrm{~mL}$ oven dried Schlenk flask was charged with $\left.600 \mathrm{mg}(0.69 \mathrm{mmol})[\mathrm{Na} \text { (diglyme })_{2}\right]\left[\mathrm{Fe}_{4} \mathrm{~N}(\mathrm{CO})_{12}\right]$ and $1.34 \mathrm{~g}(4.16 \mathrm{mmol})$ of MePTA(OTf). $30 \mathrm{~mL}$ of dry degassed THF was added to the Schlenk flask and the reaction mixture was heated at reflux for $24 \mathrm{hrs}$ under nitrogen and the reaction was monitored by IR spectroscopy. A black precipitate was observed after $10 \mathrm{~h}$. The THF was removed by filtration and the black product was washed with degassed dichloromethane $(3 \times 20 \mathrm{~mL})$. The black prepitate was then dried in vacuo. The product was purrified by crystallization from a diffusion of $\mathrm{Et}_{2} \mathrm{O}$ into a saturated $\mathrm{MeCN}$ solution at $-15^{\circ} \mathrm{C}$. X-ray quality crystals of 11-OTf $\mathrm{OT}_{3}(740 \mathrm{mg}$, $65 \%$ yield) were grown over 2 days. ${ }^{1} \mathrm{H} \mathrm{NMR}\left(400 \mathrm{MHz}, \mathrm{CD}_{3} \mathrm{CN}\right): 2.77$ (b, m, 12H, N-CH3), 3.76-5.04 (m, b, $\left.48 \mathrm{H}, \mathrm{CH}_{2}\right) .{ }^{31} \mathrm{P}\left\{{ }^{1} \mathrm{H}\right\} \mathrm{NMR}\left(400 \mathrm{MHz}, \mathrm{CD}_{3} \mathrm{CN}\right):-2.21$ (dd, JPP = 46.5, 7.5 Hz), -7.10, -16.35. ${ }^{13} \mathrm{C}$ NMR $\left(\mathrm{CD}_{3} \mathrm{CN}\right): \delta 228.07$ (s, CO), $224.44(\mathrm{~s}, \mathrm{CO}), 216.06(\mathrm{~s}, \mathrm{CO}), 120.81$, 80.54, 69.14, 61.25, 61.20, 52.31, 52.28, 52.11, 51.58, 51.53, 48.98, 48.96, 48.92, 48.90, 48.76. IR (MeCN): $v_{\mathrm{CO}}$ 1963(w), $1928(\mathrm{~s}), 1907(\mathrm{~s}), 1896(\mathrm{sh}), 1867(\mathrm{w})$. Combustion analysis calculated for $\mathrm{C}_{39} \mathrm{H}_{60} \mathrm{~F}_{9} \mathrm{Fe}_{4} \mathrm{~N}_{13} \mathrm{O}_{17} \mathrm{P}_{4} \mathrm{~S}_{3}$ : C, 29.32; H, 3.79; N, 11.40. Found: C, 29.27; H, 3.81; N, 11.29 . 
Table S1. Crystallographic data for $\left[\mathrm{Na}(\text { diglyme })_{2}\right]\left[\mathrm{Fe}_{4} \mathrm{~N}(\mathrm{CO})_{10}(\mathrm{PTA})_{2}\right]$ in $\mathrm{Na}-7$, $\left(\mathrm{Et}_{4} \mathrm{~N}\right)\left[\mathrm{Fe}_{4} \mathrm{~N}(\mathrm{CO})_{10}\left(\mathrm{PEt}_{3}\right)_{2}\right] \quad$ in $\mathrm{Et}_{4} \mathrm{~N}-8, \quad\left[\mathrm{Fe}_{4} \mathrm{~N}(\mathrm{CO})_{10}\left(\mathrm{MePTA}_{2}\right] \mathrm{I}\right.$ in 9-I and $\left[\mathrm{Fe}_{4} \mathrm{~N}(\mathrm{CO})_{8}\right.$ $\left.(\mathrm{MePTA})_{4}\right](\mathrm{OTf})_{3}$ in 11-OTf .

\begin{tabular}{|c|c|c|c|c|}
\hline & $\mathrm{Na}-\mathbf{7} \cdot \mathrm{C}_{4} \mathrm{H}_{8} \mathrm{O}$ & $\mathrm{Et}_{4} \mathrm{~N}-8$ & 9-I $\bullet\left(\mathrm{C}_{7} \mathrm{H}_{8}\right)_{1.5}$ & 11-OTf 3 \\
\hline Formula & $\begin{array}{l}{\left[\mathrm{C}_{64} \mathrm{H}_{92} \mathrm{Fe}_{8} \mathrm{~N}_{1}\right.} \\
\left.{ }_{4} \mathrm{Na}_{2} \mathrm{O}_{28} \mathrm{P}_{4}\right]\end{array}$ & $\mathrm{C}_{30} \mathrm{H}_{50} \mathrm{Fe}_{4} \mathrm{~N}_{2} \mathrm{O}_{10} \mathrm{P}_{2}$ & $\begin{array}{l}\mathrm{C}_{24} \mathrm{H}_{30} \mathrm{Fe}_{4} \mathrm{IN}_{7} \mathrm{O}_{10} \\
\mathrm{P}_{2} \bullet\left(\mathrm{C}_{7} \mathrm{H}_{8}\right)_{1.5}\end{array}$ & $\begin{array}{l}{\left[\mathrm{C}_{36} \mathrm{H}_{60} \mathrm{Fe}_{4} \mathrm{~N}_{13} \mathrm{O}_{8} \mathrm{P}_{4}\right]_{2}} \\
{\left[\mathrm{CF}_{3} \mathrm{O}_{3} \mathrm{~S}_{6}\left[\mathrm{C}_{2} \mathrm{H}_{3} \mathrm{~N}\right]_{7}\right.}\end{array}$ \\
\hline Formula wt, $\mathrm{gmol}^{-1}$ & 2122.17 & 884.06 & 1126.99 & 3482.29 \\
\hline Space group & $I 2 / \mathrm{a}$ & $\overline{P 1}$ & $\overline{P 1}$ & $\overline{P 1}$ \\
\hline$a, \AA$ & $18.2719(15)$ & $13.2981(10)$ & $9.5491(4)$ & $16.444(4)$ \\
\hline$b, \AA$ & $19.5900(14)$ & $16.8146(12)$ & $12.1879(6)$ & $19.006(4)$ \\
\hline$c, \AA$ & $22.9447(18)$ & $17.8684(13)$ & $19.0889(9)$ & $22.313(5)$ \\
\hline$\alpha, \operatorname{deg}$ & 90 & $91.0989(10)$ & $79.2142(13)$ & $90.664(4)$ \\
\hline$\beta$, deg & $96.775(2)$ & $98.3628(11)$ & $85.3076(13)$ & $97.383(3)$ \\
\hline$\gamma, \operatorname{deg}$ & 90 & $95.6962(11)$ & $79.4246(14)$ & $90.103(4)$ \\
\hline$V, \AA^{3}$ & $8155.6(11)$ & $3928.5(5)$ & $2142.72(17)$ & $6962(3)$ \\
\hline$Z$ & 4 & 4 & 1 & 2 \\
\hline$T, \mathrm{~K}$ & $100(2)$ & $90(2)$ & 90 & $100(2)$ \\
\hline$\rho$, calcd, $\mathrm{g} \mathrm{cm}^{-3}$ & 1.728 & 1.495 & 1.747 & 1.672 \\
\hline Refl. collected $/ 2 \theta_{\max }$ & $27720 / 55.286$ & $46915 / 61.29$ & $28178 / 136.48$ & $47553 / 50.50$ \\
\hline Uniq refl./ $I>2 \sigma(I)$ & $9394 / 6756$ & $24092 / 20874$ & $7534 / 7161$ & $24931 / 18307$ \\
\hline No. param/restrains & $558 / 55$ & $1062 / 155$ & $591 / 48$ & $1784 / 0$ \\
\hline$\lambda, \mathrm{A}^{\circ} / \mu(\mathrm{K} \alpha), \mathrm{cm}^{-1}$ & 0.71073 & 0.71073 & 1.54 & 0.71073 \\
\hline $\mathrm{R}_{1} / \mathrm{GOF}$ & $0.0609 / 1.026$ & $0.0295 / 1.030$ & $0.0625 / 1.051$ & $0.0841 / 1.081$ \\
\hline $\mathrm{wR}_{2}(I>2 \sigma(I))^{a}$ & 0.1492 & 0.0532 & 0.1904 & 0.1939 \\
\hline Res. density, e $\AA^{-3}$ & $1.930 /-0.924$ & $0.504 /-0.532$ & $3.413 /-1.578$ & $1.618 /-1.246$ \\
\hline
\end{tabular}

$$
{ }^{a} R_{1}=\Sigma\left\|F_{\mathrm{o}}\left|-F_{\mathrm{c}} \| / \Sigma\right| F_{\mathrm{o}} \mid, \mathrm{w} R_{2}=\left\{\Sigma\left[\mathrm{w}\left(F_{\mathrm{o}}^{2}-F_{\mathrm{c}}^{2}\right)^{2}\right] / \Sigma\left[\mathrm{w}\left(F_{\mathrm{o}}^{2}\right)^{2}\right]\right\}^{0.5}\right.
$$


Table S2. Selected average interatomic distances $(\AA)$ and selected average angles (deg) for $\left.[\mathrm{Na} \text { (diglyme })_{2}\right]\left[\mathrm{Fe}_{4} \mathrm{~N}(\mathrm{CO})_{10}(\mathrm{PTA})_{2}\right]$ in Na-7, $\left(\mathrm{Et}_{4} \mathrm{~N}\right)\left[\mathrm{Fe}_{4} \mathrm{~N}(\mathrm{CO})_{10}\left(\mathrm{PEt}_{3}\right)_{2}\right]$ in $\mathrm{Et}_{4} \mathrm{~N}-8$, $\left[\mathrm{Fe}_{4} \mathrm{~N}(\mathrm{CO})_{10}\left({ }^{\mathrm{MePTA}}\right)_{2}\right] \mathrm{I}$ in 9-I, $\left[\mathrm{Fe}_{4} \mathrm{~N}(\mathrm{CO})_{8}\left({ }^{\mathrm{MePTA}}\right)_{4}\right](\mathrm{OTf})_{3}$ in 11-OTf 3 and previously reported $\left[\mathrm{Et}_{4} \mathrm{~N}\right]\left[\mathrm{Fe}_{4} \mathrm{~N}(\mathrm{CO})_{12}\right]$ in $\mathrm{Et}_{4} \mathrm{~N}-1 .{ }^{8}$ See Chart $\mathrm{S} 1$ for atom numbering scheme.

\begin{tabular}{cccccc}
\hline & $\mathrm{Et}_{4} \mathrm{~N}-1$ & $\mathrm{Na}-7$ & $\mathrm{Et}_{4} \mathrm{~N}-8$ & $9-\mathrm{I}$ & $11-\mathrm{OTf}$ \\
\hline $\mathrm{Fe}_{1}-\mathrm{N}$ & $1.768(3)$ & $1.782(4)$ & $1.783(1)$ & $1.768(4)$ & $1.779(5)$ \\
$\mathrm{Fe}_{2}-\mathrm{N}$ & $1.896(3)$ & $1.924(4)$ & $1.923(2)$ & $1.936(4)$ & $1.925(6)$ \\
$\mathrm{Fe}_{3}-\mathrm{N}$ & $1.909(3)$ & $1.910(4)$ & $1.935(1)$ & $1.909(4)$ & $1.890(5)$ \\
$\mathrm{Fe}_{4}-\mathrm{N}$ & $1.775(3)$ & $1.777(4)$ & $1.780(1)$ & $1.762(4)$ & $1.780(5)$ \\
$\mathrm{Fe}_{1}-\mathrm{Fe}_{2}$ & $2.6186(7)$ & $2.5933(10)$ & $2.6187(5)$ & $2.5981(10)$ & $2.626(1)$ \\
$\mathrm{Fe}_{1}-\mathrm{Fe}_{3}$ & $2.6005(7)$ & $2.5827(10)$ & $2.5935(5)$ & $2.6137(10)$ & $2.624(1)$ \\
$\mathrm{Fe}_{2}-\mathrm{Fe}_{3}$ & $2.5065(7)$ & $2.5065(10)$ & $2.4985(4)$ & $2.5006(11)$ & $2.509(1)$ \\
$\mathrm{Fe}_{2}-\mathrm{Fe}_{4}$ & $2.6147(7)$ & $2.5881(10)$ & $2.6065(5)$ & $2.6317(10)$ & $2.614(1)$ \\
$\mathrm{Fe}_{3}-\mathrm{Fe}_{4}$ & $2.5916(7)$ & $2.6048(9)$ & $2.6177(5)$ & $2.5889(10)$ & $2.608(1)$ \\
$\mathrm{Fe}_{1}-\mathrm{P}$ & -- & $2.1793(13)$ & $2.2189(5)$ & $2.1553(13)$ & $2.170(2)$ \\
& & & & & $2.211(2)$ \\
$\mathrm{Fe}_{4}-\mathrm{P}$ & -- & $2.1726(14)$ & $2.2155(5)$ & $2.1516(17)$ & $2.158(2)$ \\
$\mathrm{Fe}_{3}-\mathrm{P}$ & -- & -- & -- & -- & $2.187(2)$ \\
$<\mathrm{Fe}_{2}-\mathrm{Fe}_{1}-\mathrm{Fe}_{3}>$ & $57.40(2)$ & $57.93(3)$ & $60.85(1)$ & $57.34(3)$ & $57.09(4)$ \\
$<\mathrm{Fe}_{1}-\mathrm{Fe}_{2}-\mathrm{Fe}_{4}>$ & $85.19(2)$ & $86.73(3)$ & $85.96(1)$ & $84.90(3)$ & $85.53(4)$ \\
$<\mathrm{Fe}_{1}-\mathrm{Fe}_{3}-\mathrm{Fe}_{4}>$ & $86.02(2)$ & $86.60(3)$ & $86.24(1)$ & $85.45(3)$ & $85.69(4)$ \\
$<\mathrm{Fe}_{1}-\mathrm{N}_{1}-\mathrm{Fe}_{4}>$ & $177.9(3)$ & $177.3(2)$ & $177.82(6)$ & $179.6(3)$ & $178.1(3)$ \\
\hline & & & & &
\end{tabular}


Chart S1. Atom numbering schemes for Table S2.
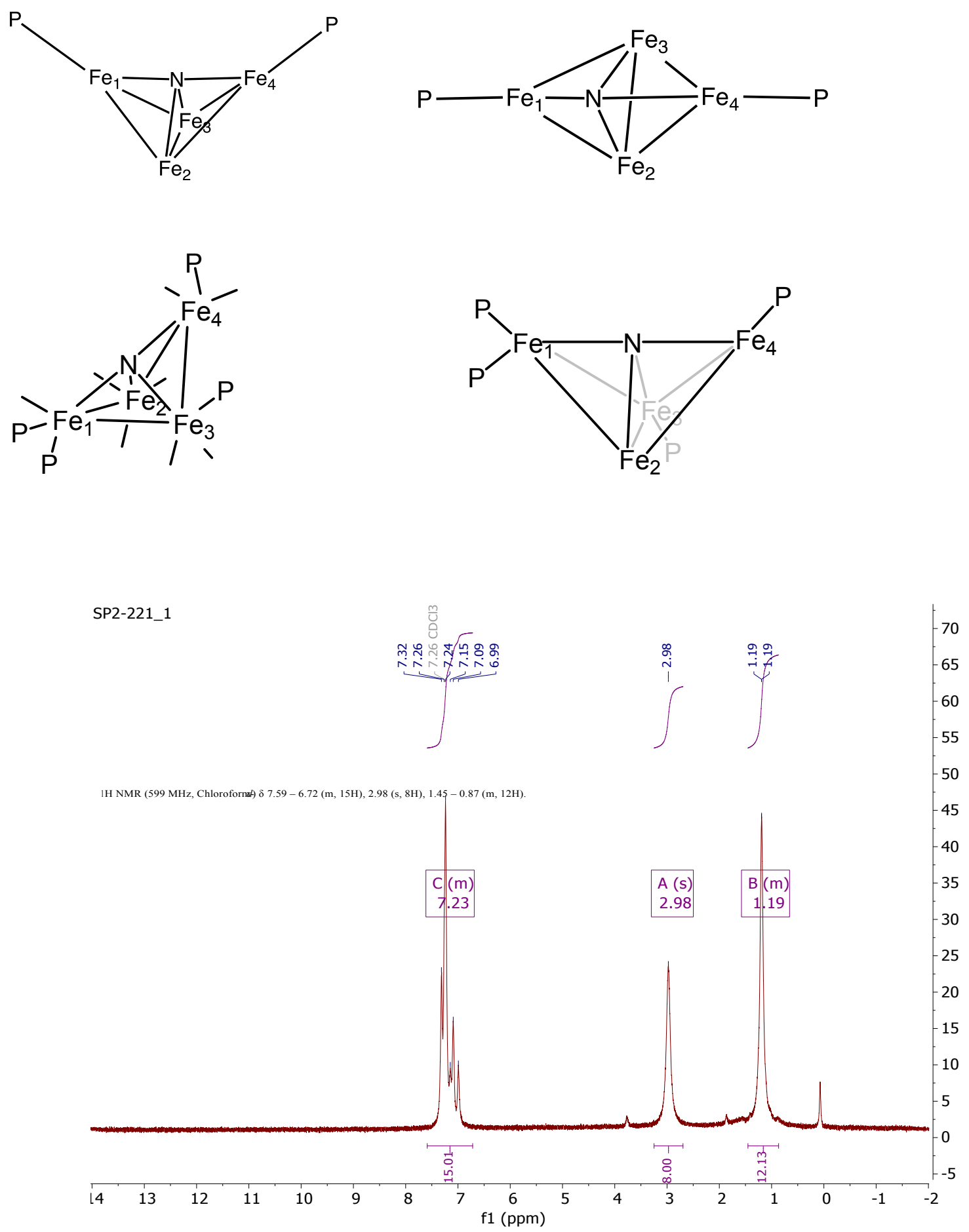

Figure S1. ${ }^{1} \mathrm{H}$ NMR $\left(\mathrm{CDCl}_{3}, 298 \mathrm{~K}, 400 \mathrm{MHz}\right)$ of $\mathrm{Et}_{4} \mathrm{~N}-2$. 


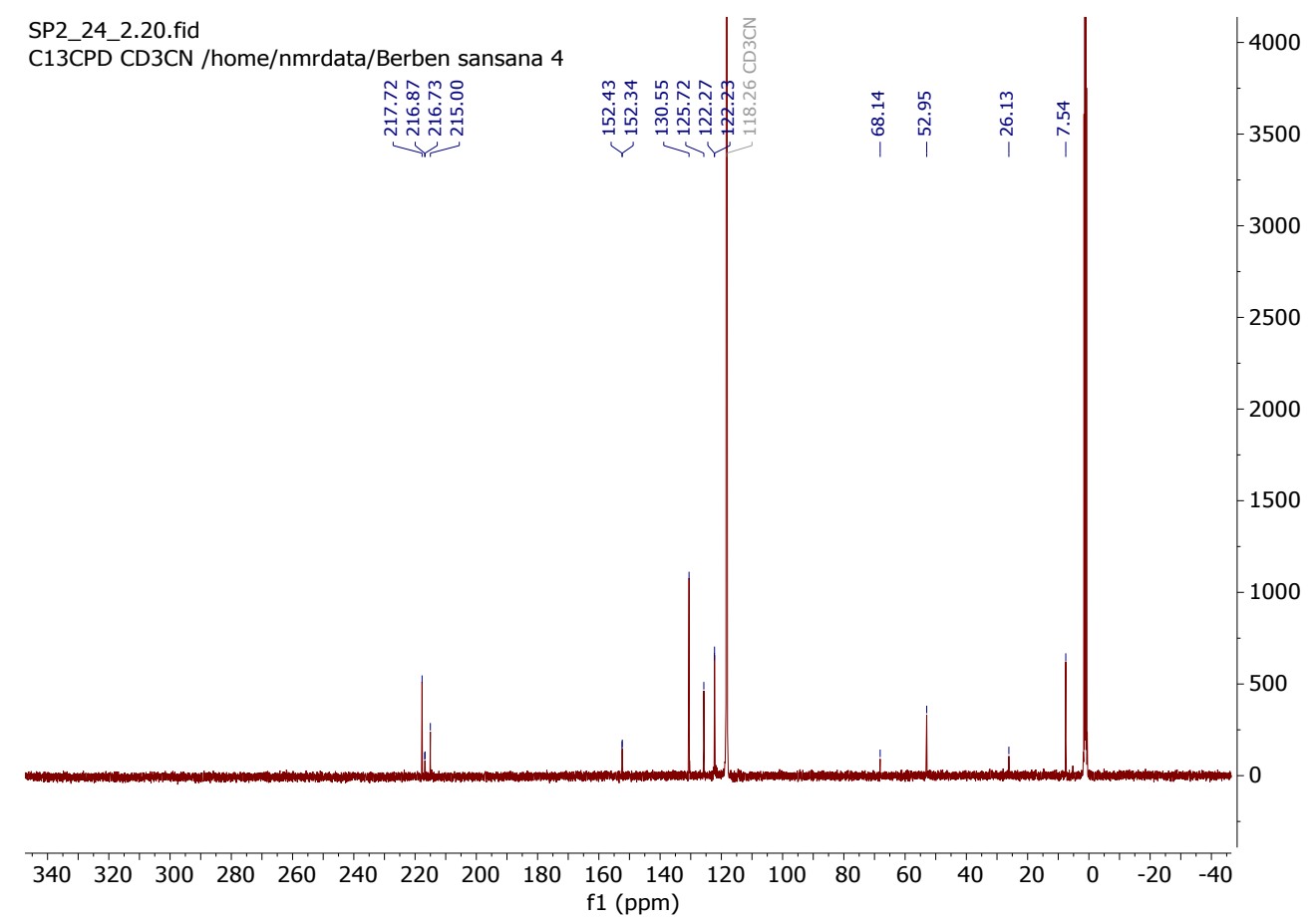

Figure S2. ${ }^{13} \mathrm{C} \mathrm{NMR}\left(\mathrm{CD}_{3} \mathrm{CN}, 298 \mathrm{~K}, 400 \mathrm{MHz}\right)$ of $\mathrm{Et}_{4} \mathrm{~N}-2$.

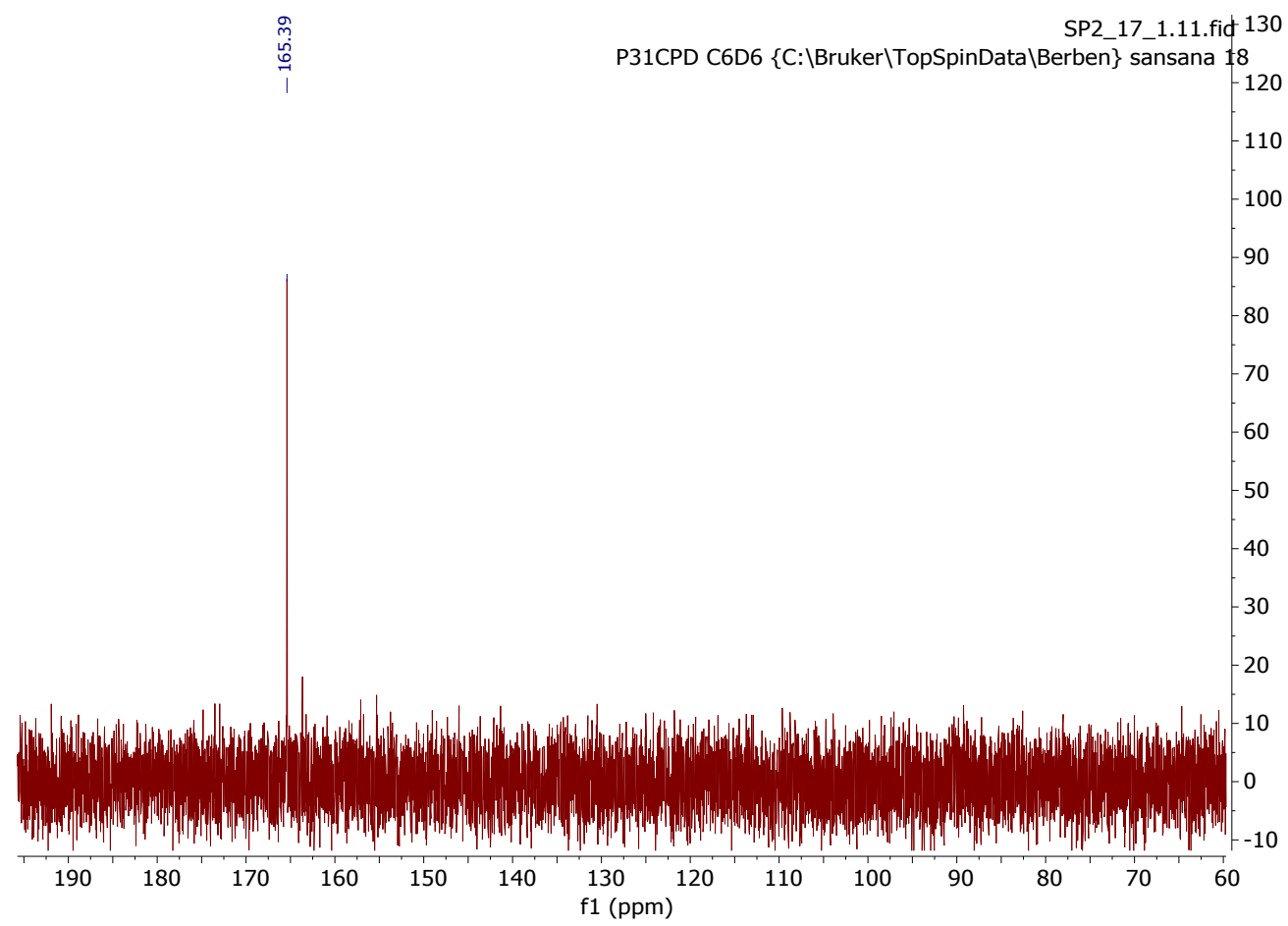

Figure S3. ${ }^{31} \mathrm{P}\left\{{ }^{1} \mathrm{H}\right\} \mathrm{NMR}\left(\mathrm{C}_{6} \mathrm{D}_{6}, 298 \mathrm{~K}, 400 \mathrm{MHz}\right)$ of $\mathrm{Et}_{4} \mathrm{~N}-2$. 


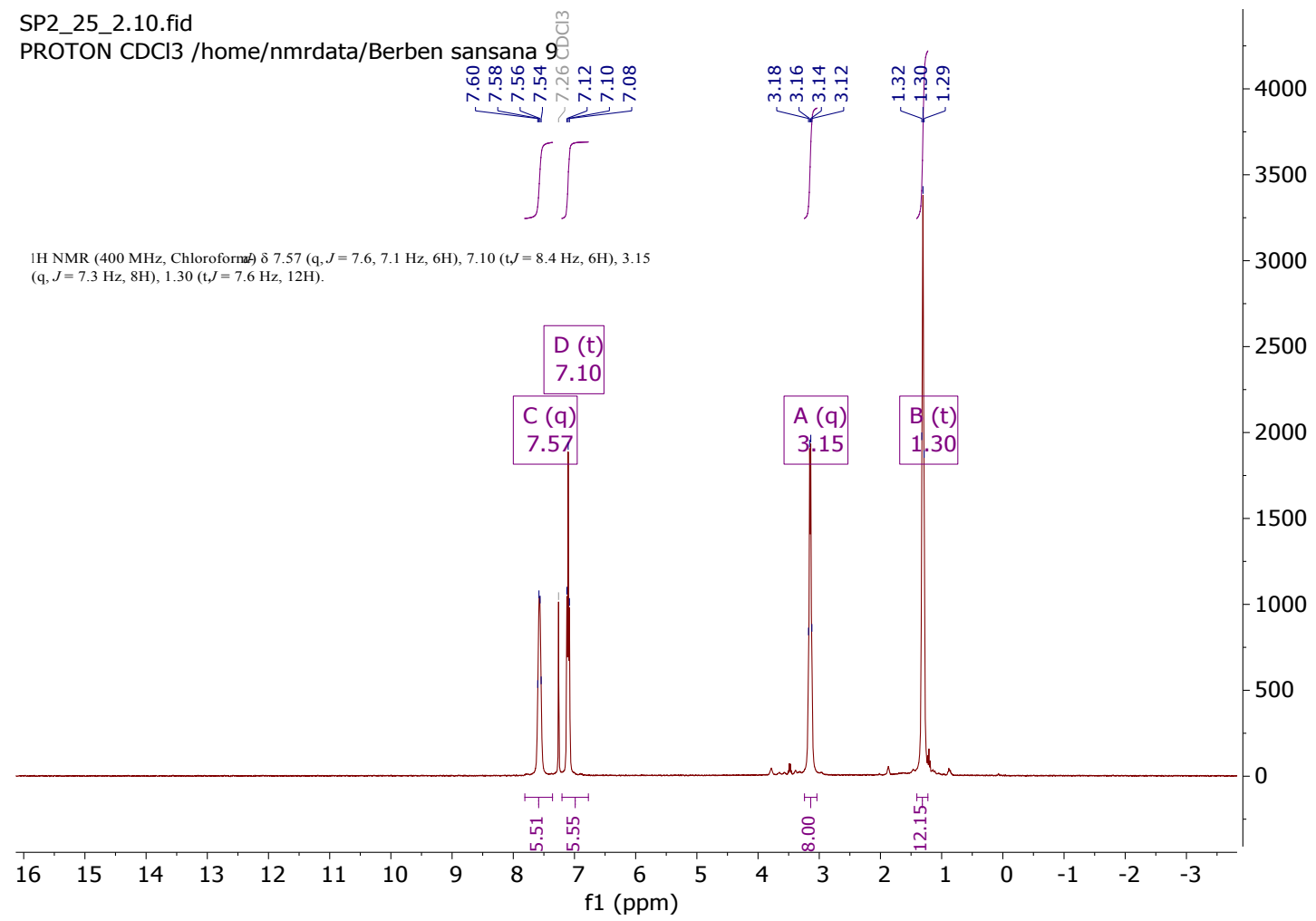

Figure S4. ${ }^{1} \mathrm{H} \mathrm{NMR}\left(\mathrm{CDCl}_{3}, 298 \mathrm{~K}, 400 \mathrm{MHz}\right)$ of $\mathrm{Et}_{4} \mathrm{~N}-3$.

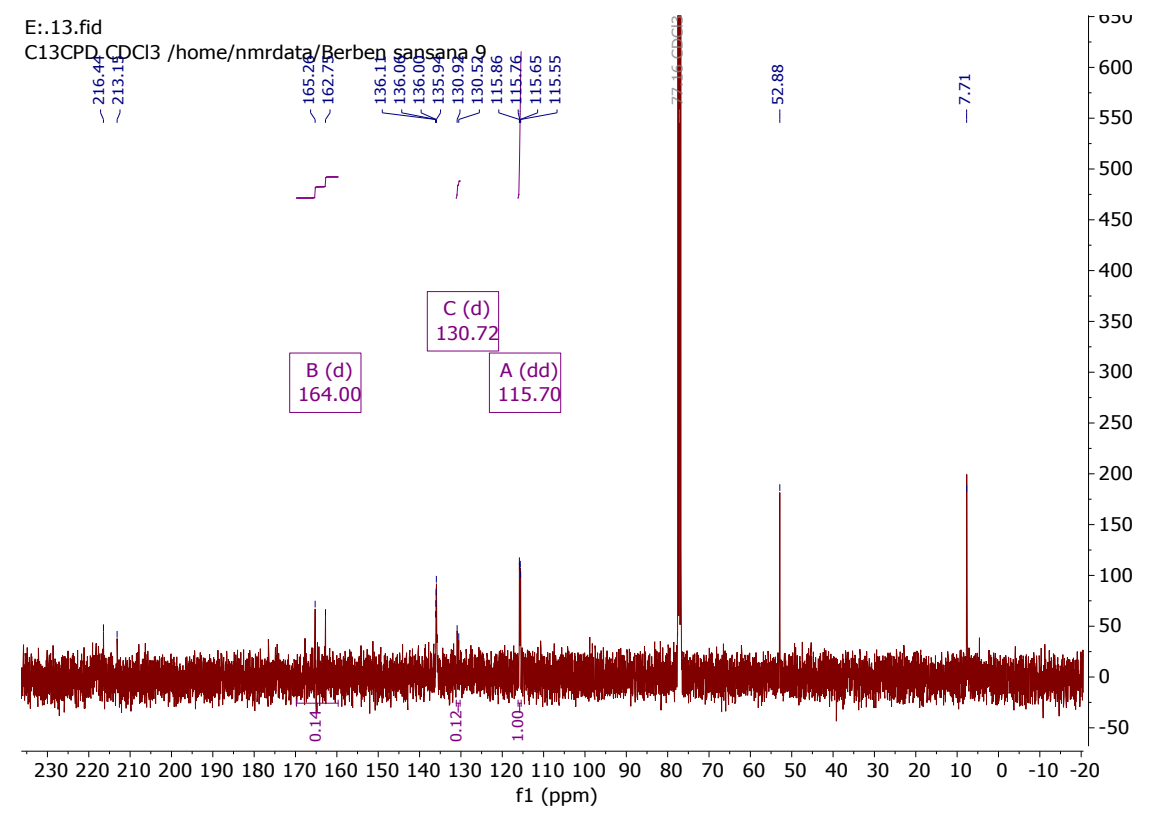

Figure S5. ${ }^{13} \mathrm{C}$ NMR $\left(\mathrm{CDCl}_{3}, 298 \mathrm{~K}, 400 \mathrm{MHz}\right)$ of $\mathrm{Et}_{4} \mathrm{~N}-3$. 


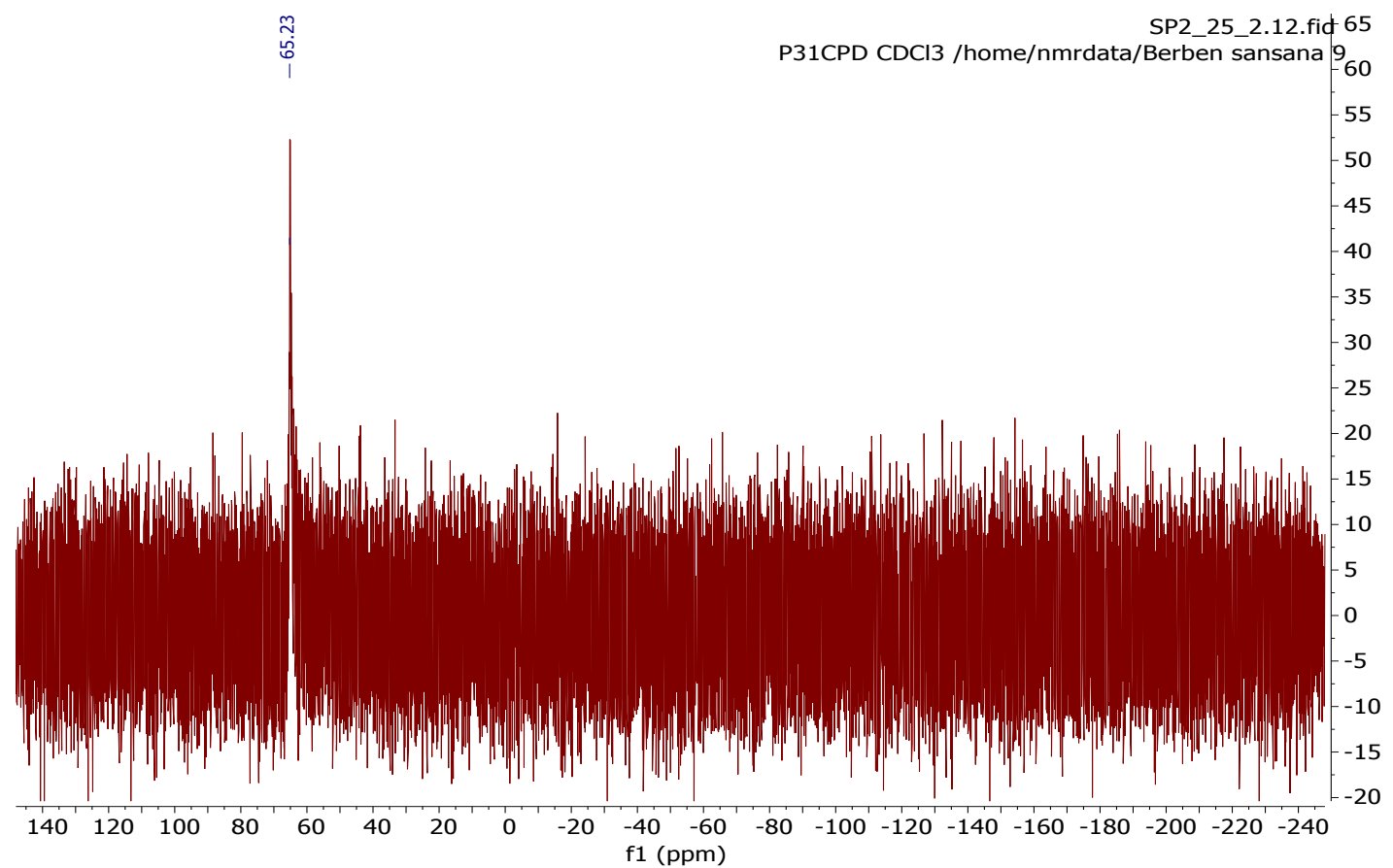

Figure S6. ${ }^{31} \mathrm{P}\left\{{ }^{1} \mathrm{H}\right\} \mathrm{NMR}\left(\mathrm{CDCl}_{3}, 298 \mathrm{~K}, 400 \mathrm{MHz}\right)$ of $\mathrm{Et}_{4} \mathrm{~N}-3$.

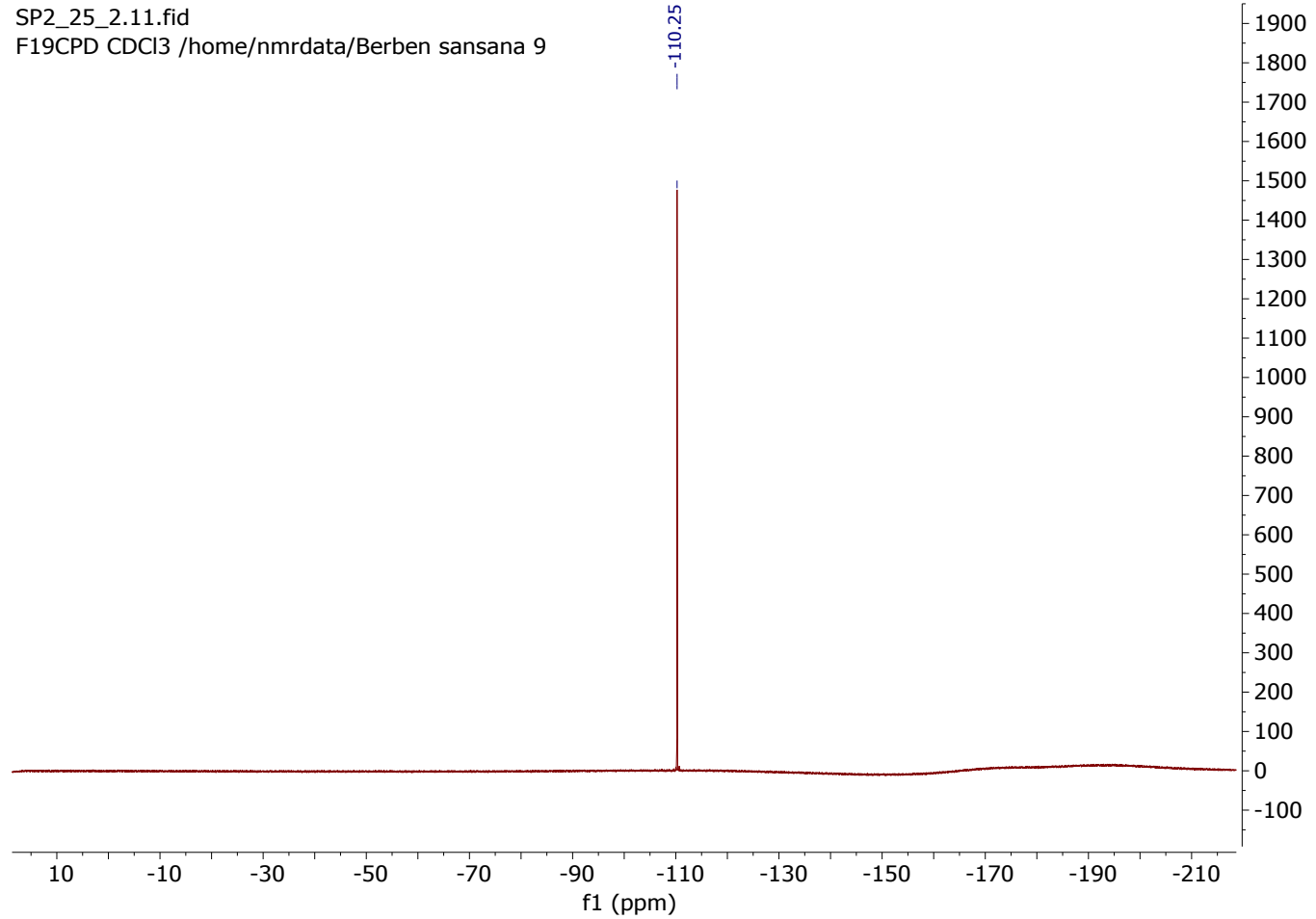

Figure S7. ${ }^{19} \mathrm{~F}$ NMR $\left(\mathrm{CDCl}_{3}, 298 \mathrm{~K}, 400 \mathrm{MHz}\right)$ of $\mathrm{Et}_{4} \mathrm{~N}-3$. 


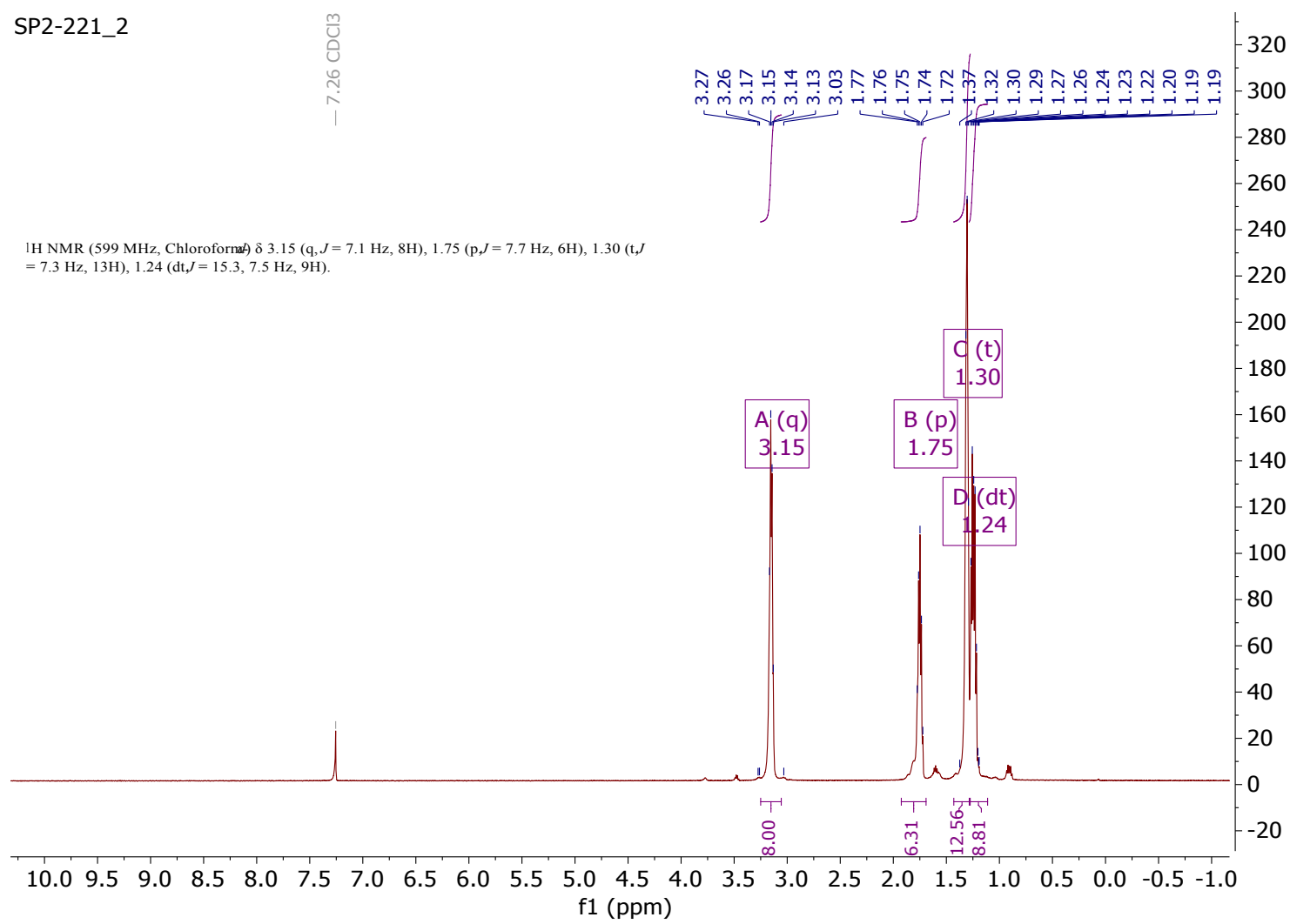

Figure S8. ${ }^{1} \mathrm{H}$ NMR $\left(\mathrm{C}_{6} \mathrm{D}_{6}, 298 \mathrm{~K}, 400 \mathrm{MHz}\right)$ of $\mathrm{Et}_{4} \mathrm{~N}-4$.

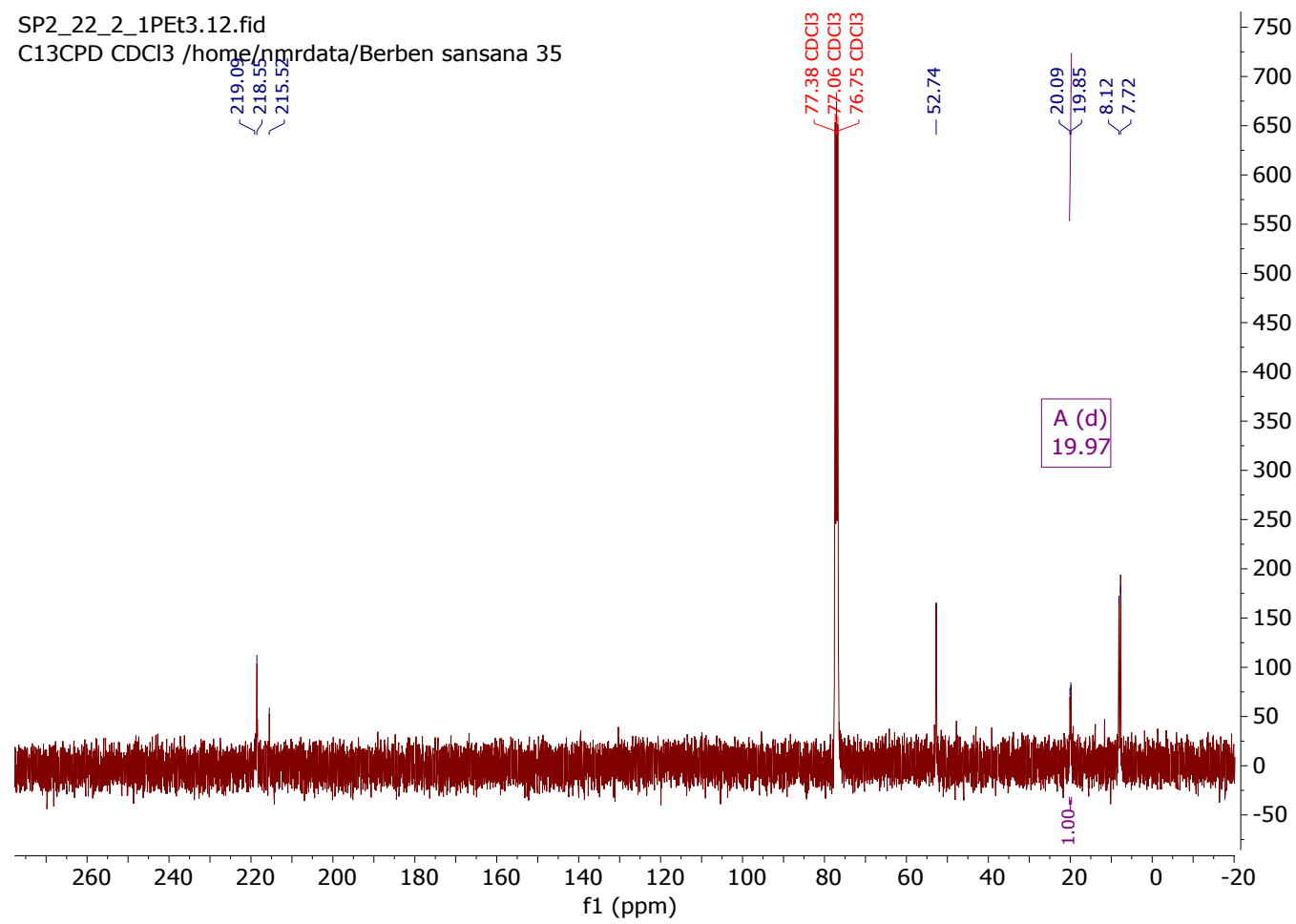

Figure S9. ${ }^{13} \mathrm{C} \mathrm{NMR}\left(\mathrm{CDCl}_{3}, 298 \mathrm{~K}, 400 \mathrm{MHz}\right)$ of $\mathrm{Et}_{4} \mathrm{~N}-4$. 


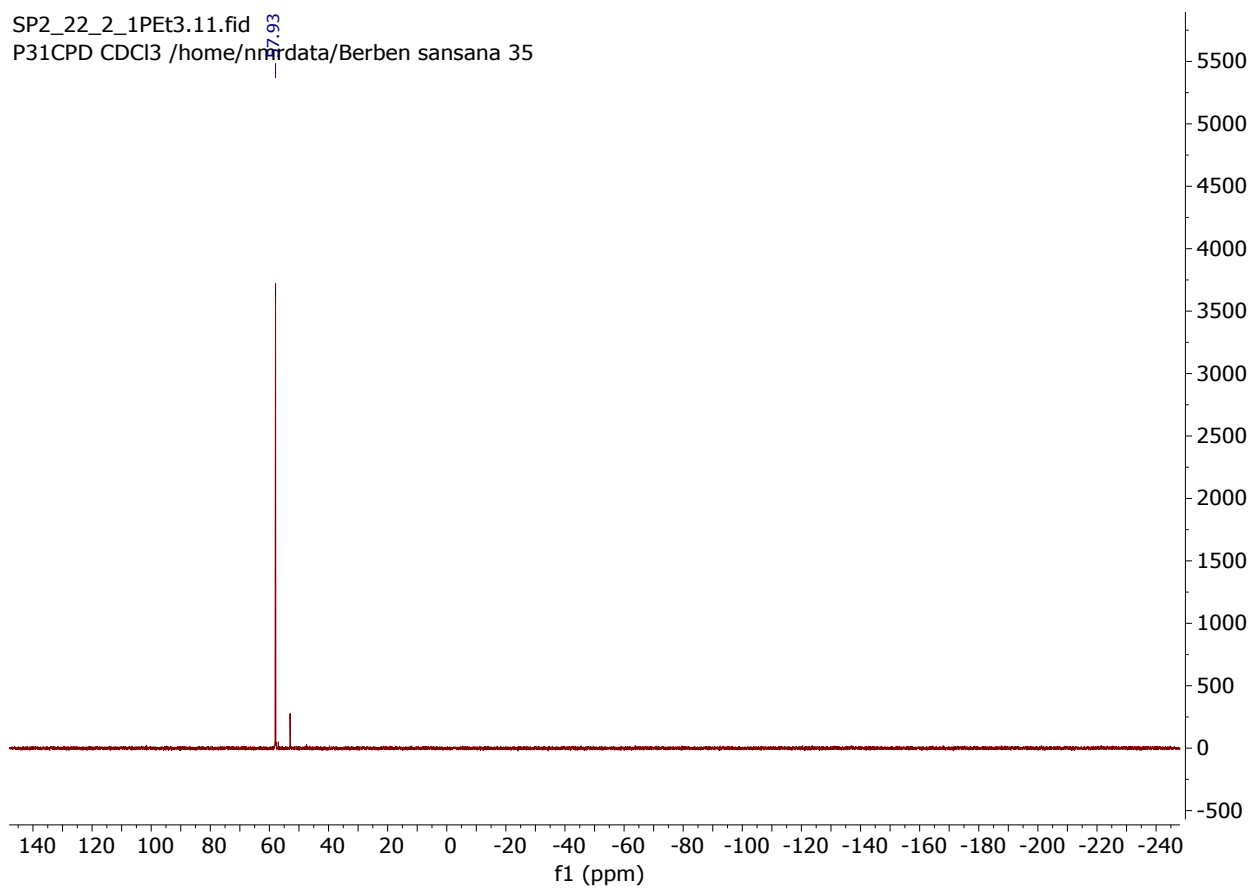

Figure S10. ${ }^{31} \mathrm{P}\left\{{ }^{1} \mathrm{H}\right\} \mathrm{NMR}\left(\mathrm{CDCl}_{3}, 298 \mathrm{~K}, 400 \mathrm{MHz}\right)$ of $\mathrm{Et}_{4} \mathrm{~N}-4$.

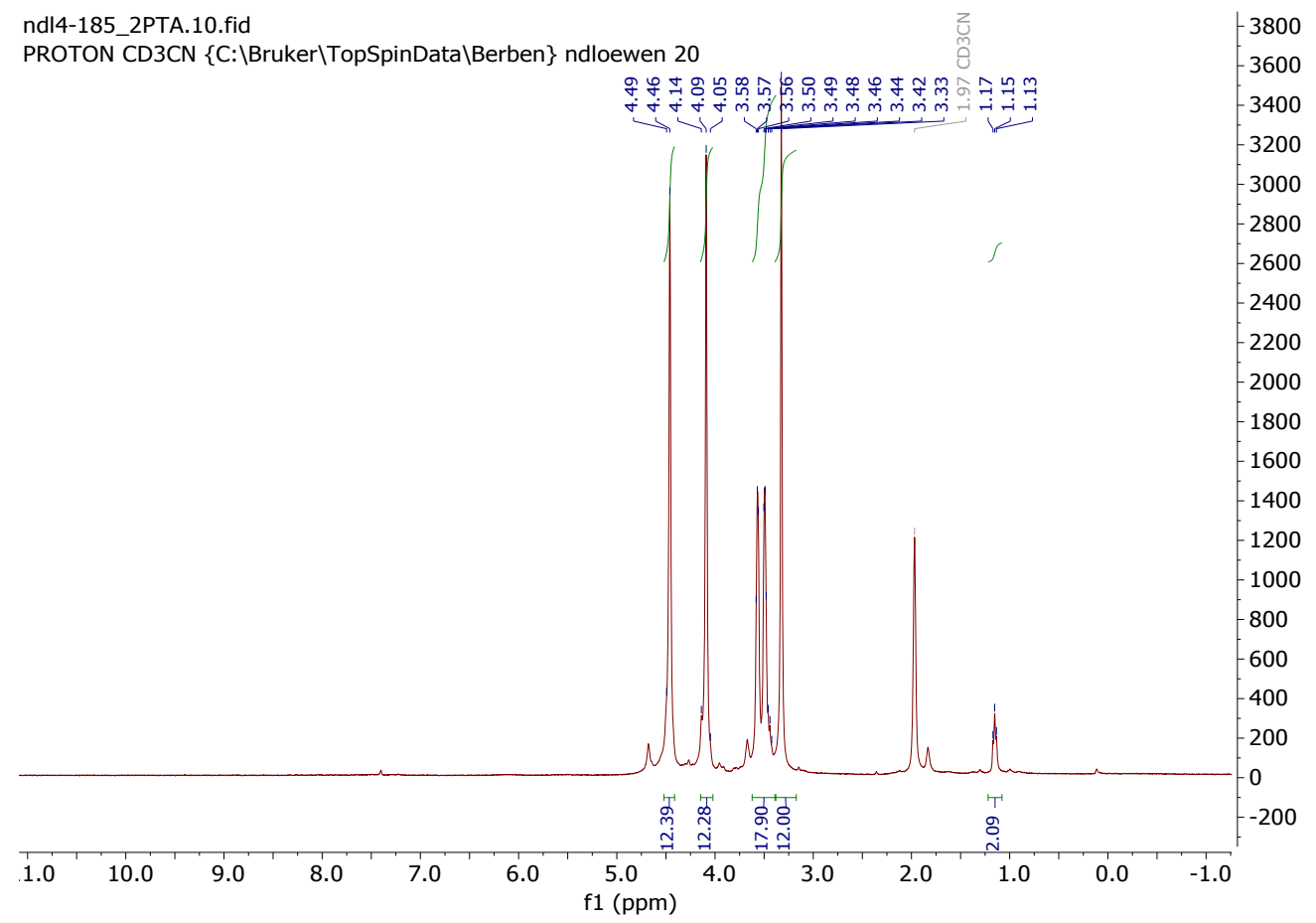

Figure S11. ${ }^{1} \mathrm{H}$ NMR $\left(\mathrm{CD}_{3} \mathrm{CN}, 298 \mathrm{~K}, 400 \mathrm{MHz}\right)$ of Na-7. 


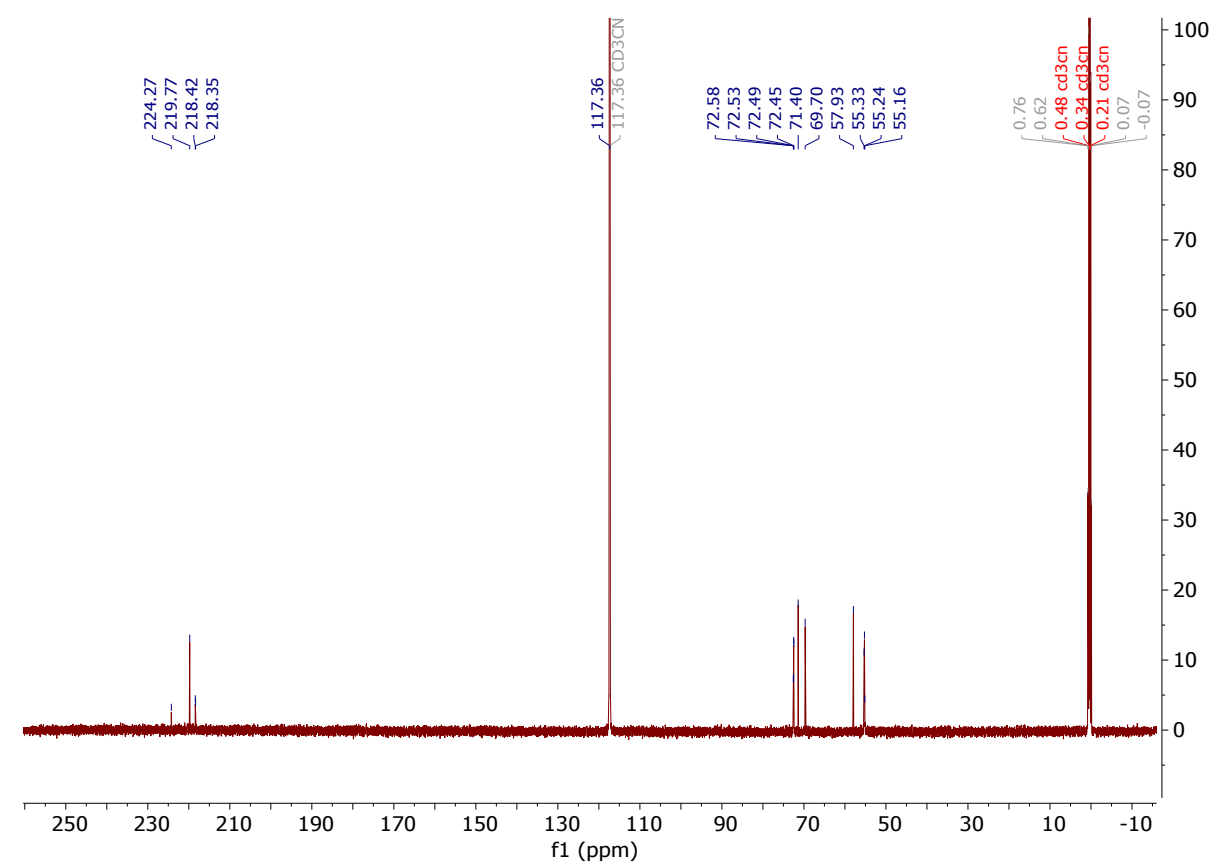

Figure S12. ${ }^{13} \mathrm{C}$ NMR $\left(\mathrm{CD}_{3} \mathrm{CN}, 298 \mathrm{~K}, 600 \mathrm{MHz}\right)$ of Na-7.

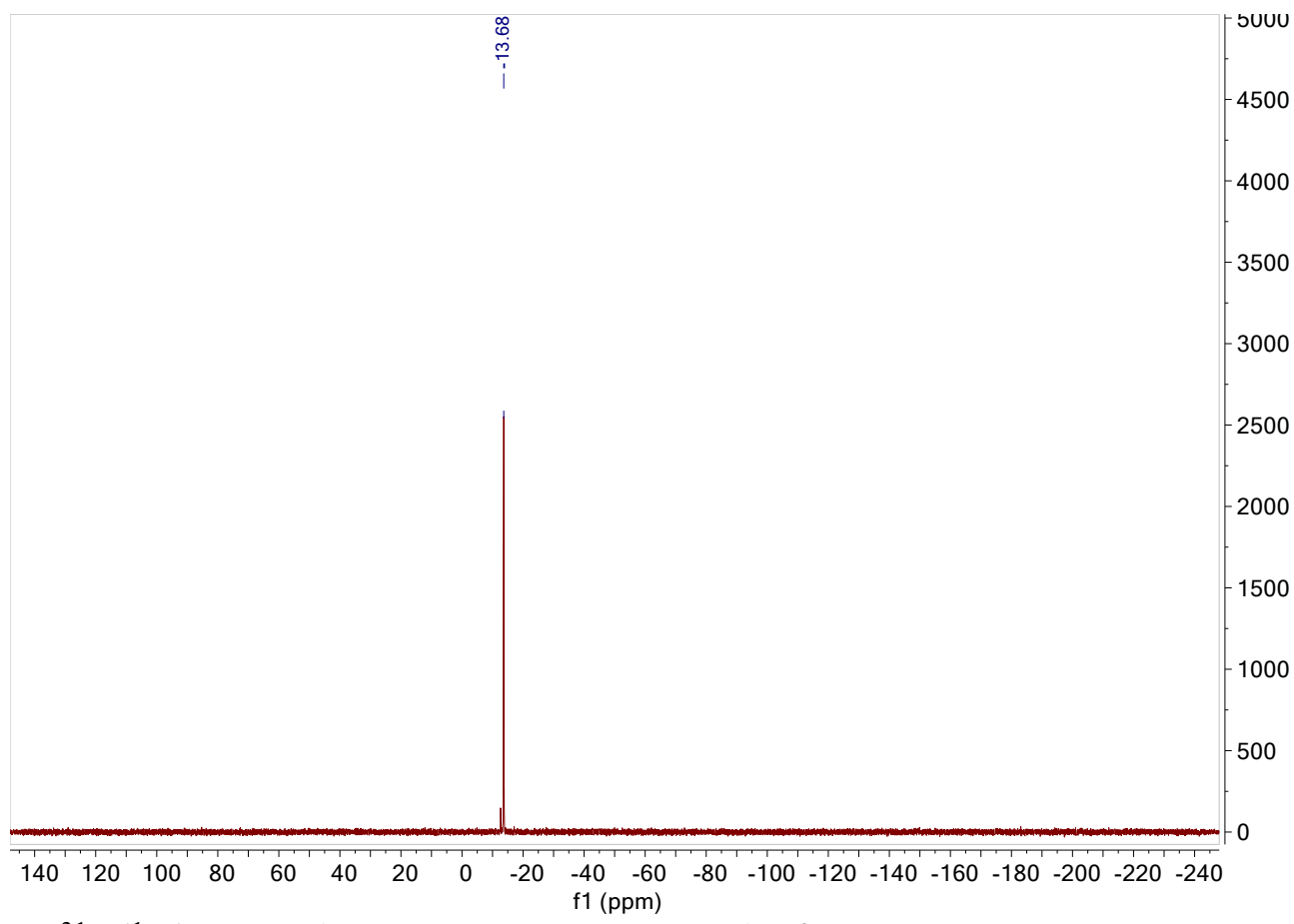

Figure S13. ${ }^{31} \mathrm{P}\left\{{ }^{1} \mathrm{H}\right\} \mathrm{NMR}\left(\mathrm{CD}_{3} \mathrm{CN}, 298 \mathrm{~K}, 400 \mathrm{MHz}\right)$ of $\mathrm{Na}-7$. 


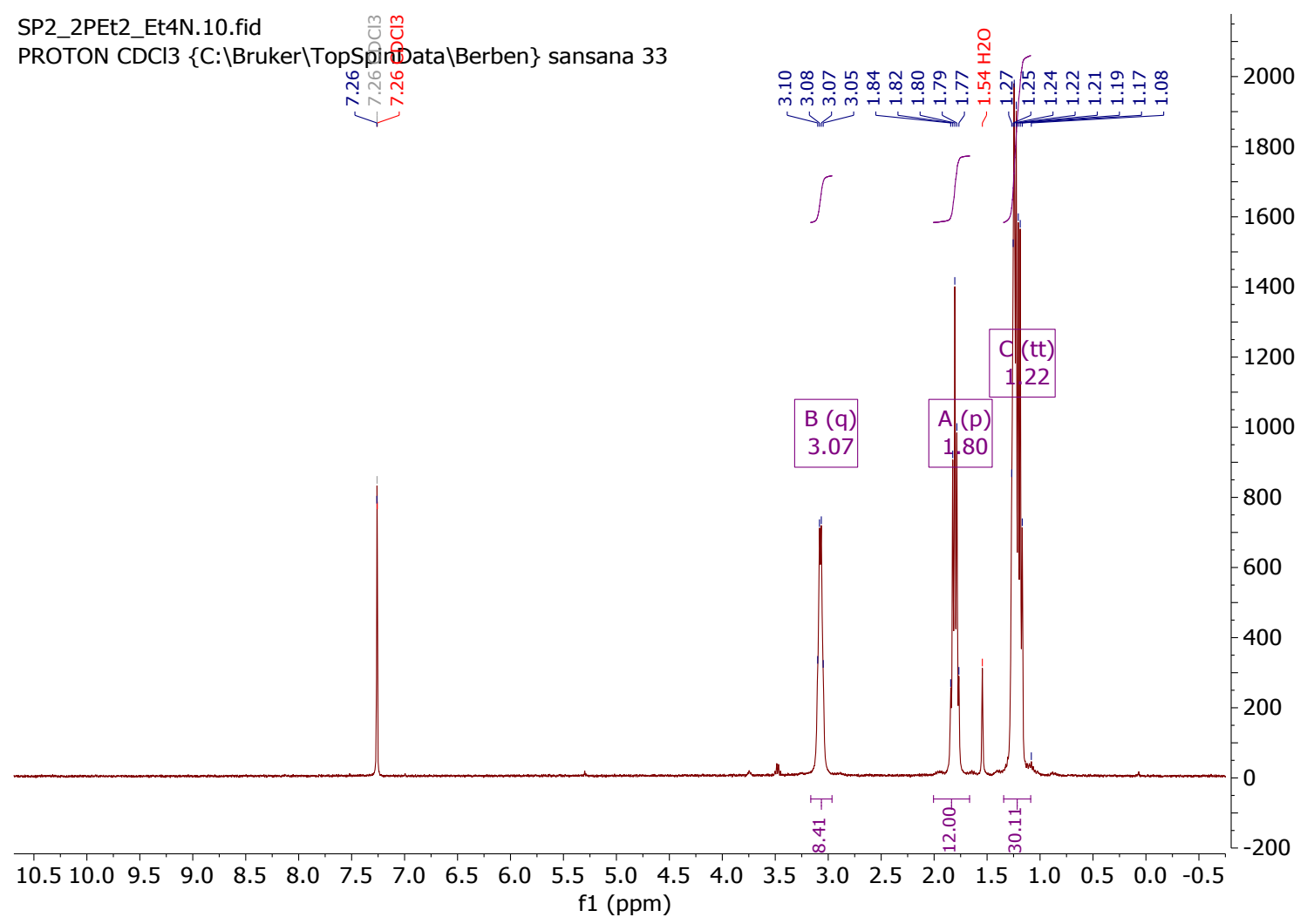

Figure S14. ${ }^{1} \mathrm{H} \mathrm{NMR}\left(\mathrm{CDCl}_{3}, 298 \mathrm{~K}, 400 \mathrm{MHz}\right)$ of $\mathrm{Et}_{4} \mathrm{~N}-8$.

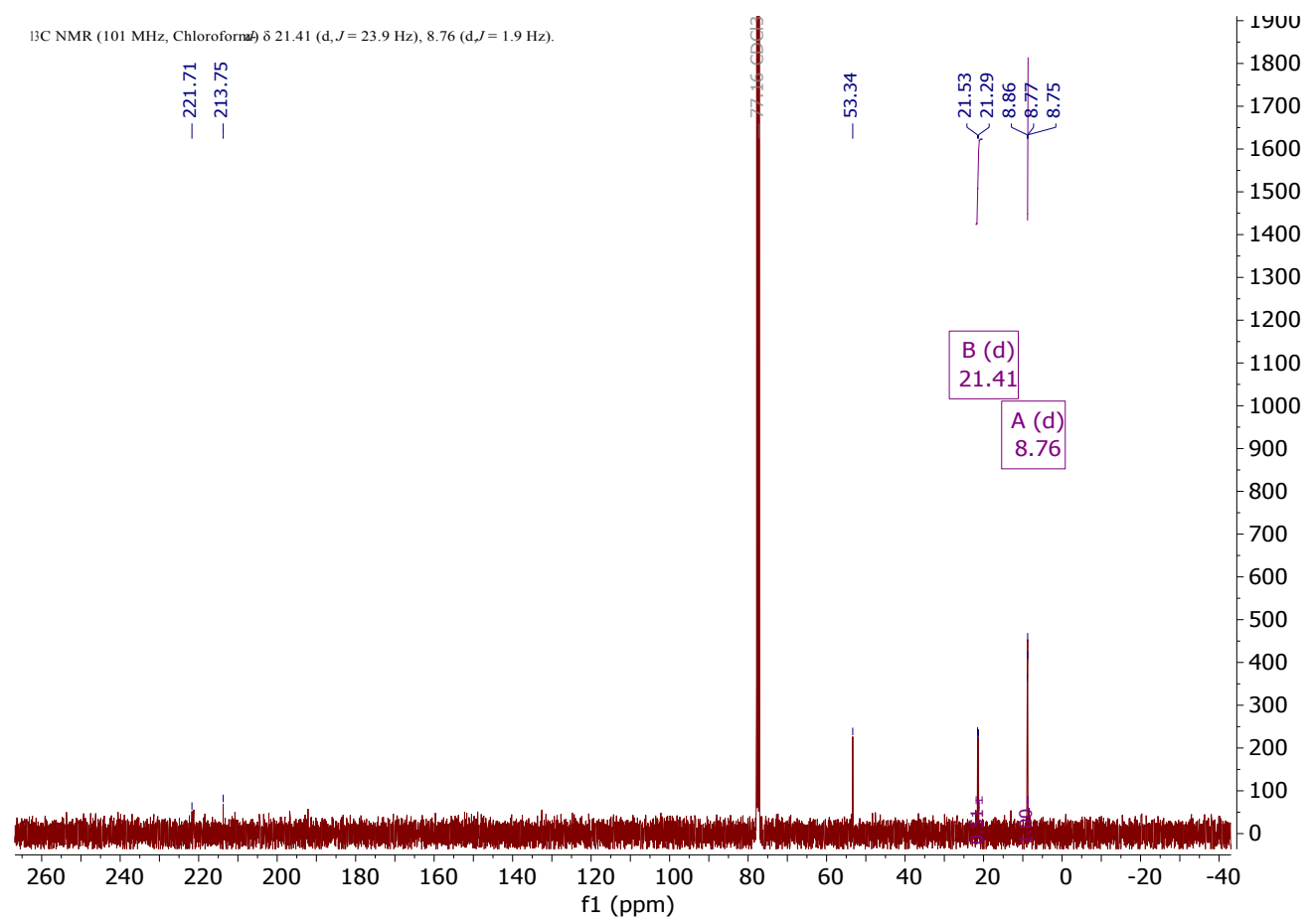

Figure S15. ${ }^{13} \mathrm{C}$ NMR $\left(\mathrm{CDCl}_{3}, 298 \mathrm{~K}, 400 \mathrm{MHz}\right)$ of $\mathrm{Et}_{4} \mathrm{~N}-8$. 


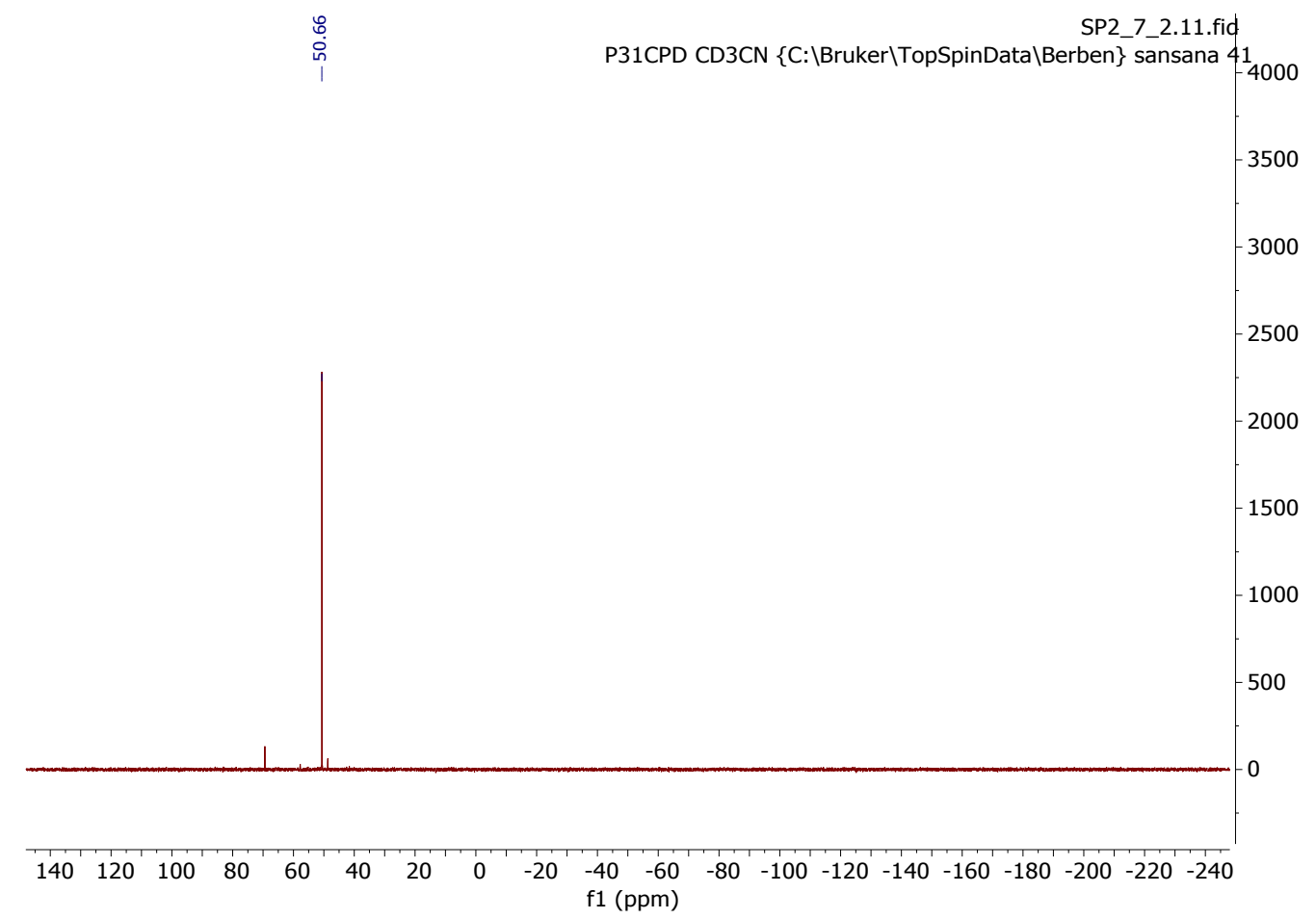

Figure S16. ${ }^{31} \mathrm{P}\left\{{ }^{1} \mathrm{H}\right\} \mathrm{NMR}\left(\mathrm{CDCl}_{3}, 298 \mathrm{~K}, 400 \mathrm{MHz}\right)$ of $\mathrm{Et}_{4} \mathrm{~N}-8$.

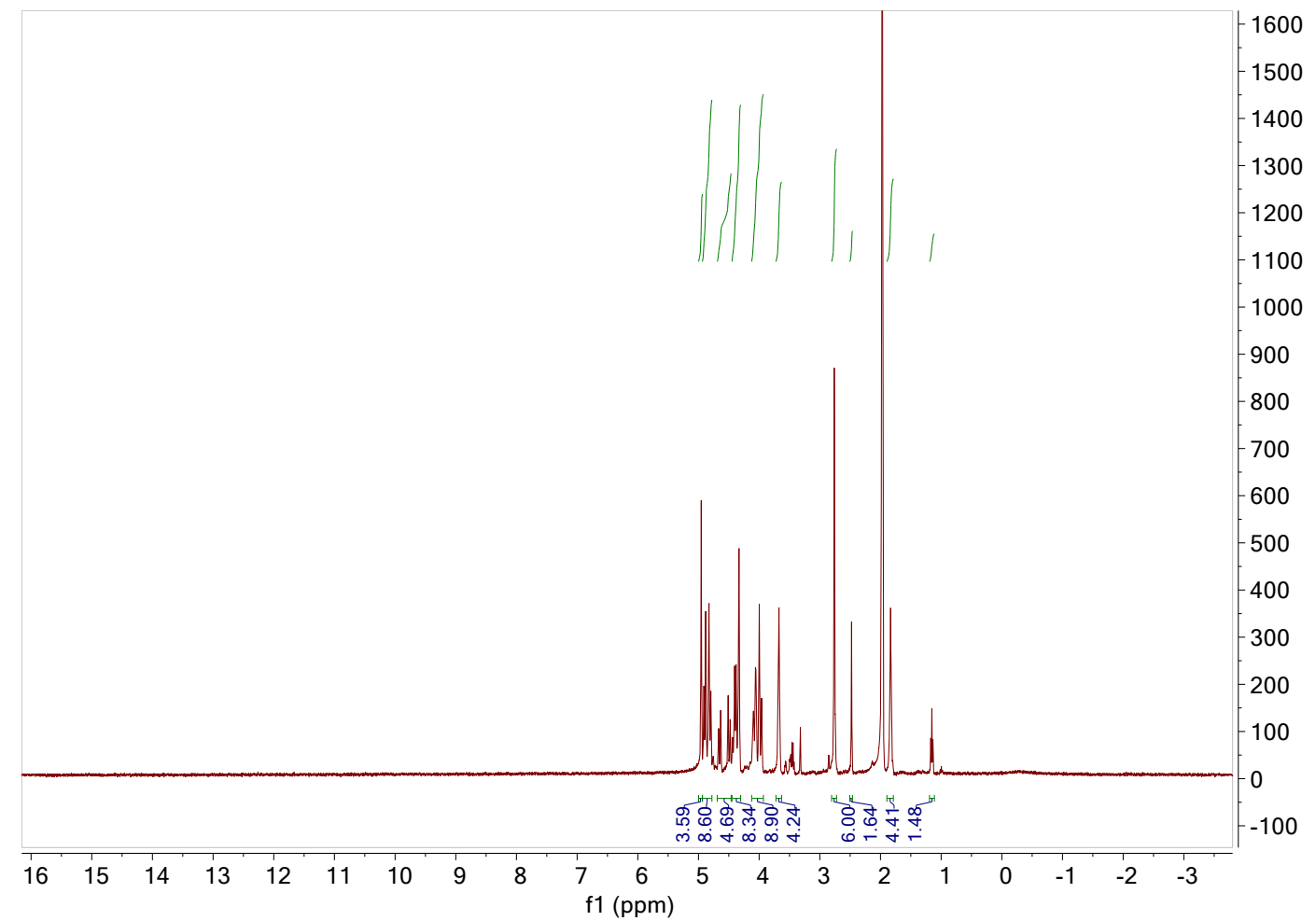

Figure S17. ${ }^{1} \mathrm{H}$ NMR $\left(\mathrm{CD}_{3} \mathrm{CN}, 298 \mathrm{~K}, 400 \mathrm{MHz}\right)$ of 9-OTf. 


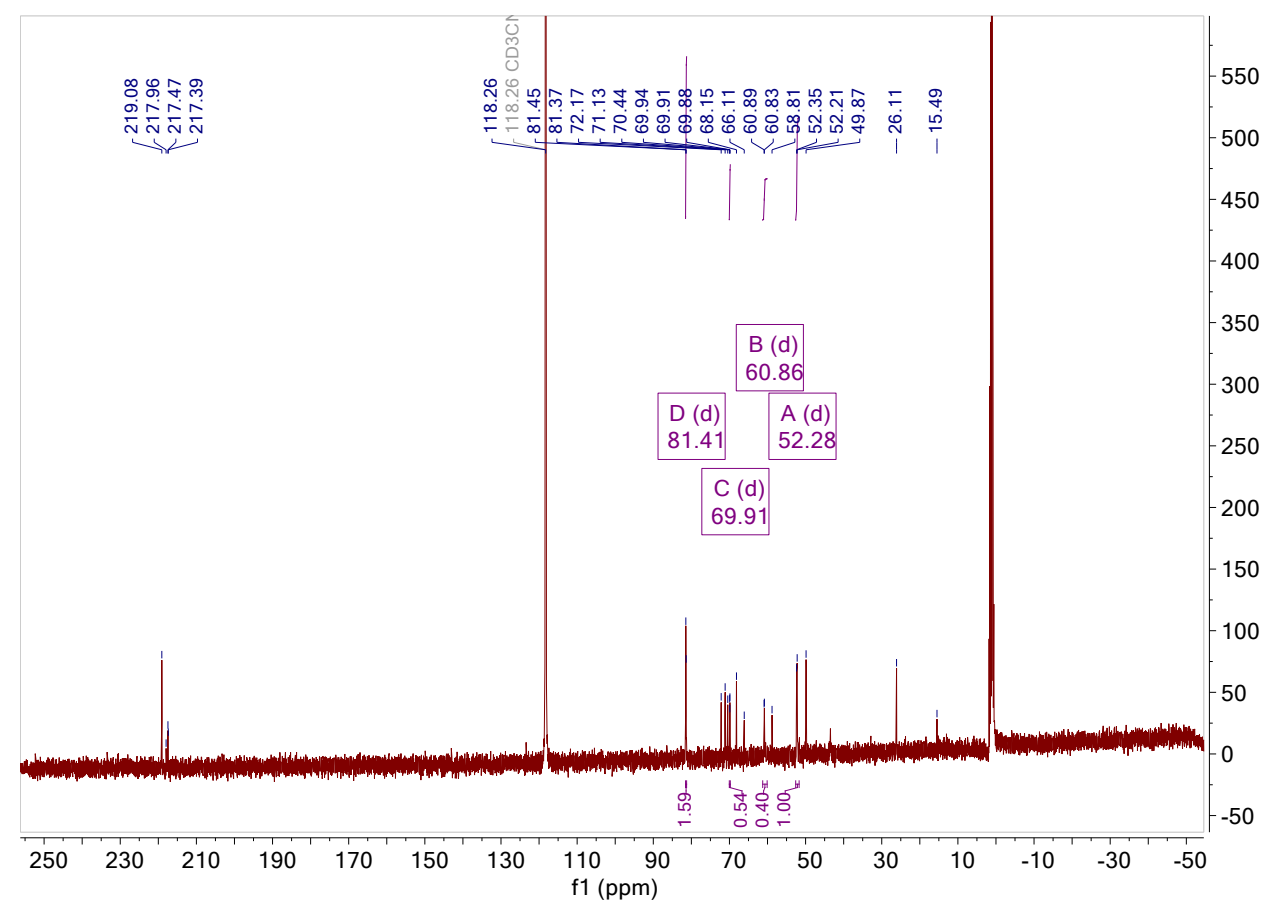

Figure S18. ${ }^{13} \mathrm{C}$ NMR $\left(\mathrm{CD}_{3} \mathrm{CN}, 298 \mathrm{~K}, 400 \mathrm{MHz}\right)$ of 9-OTf.

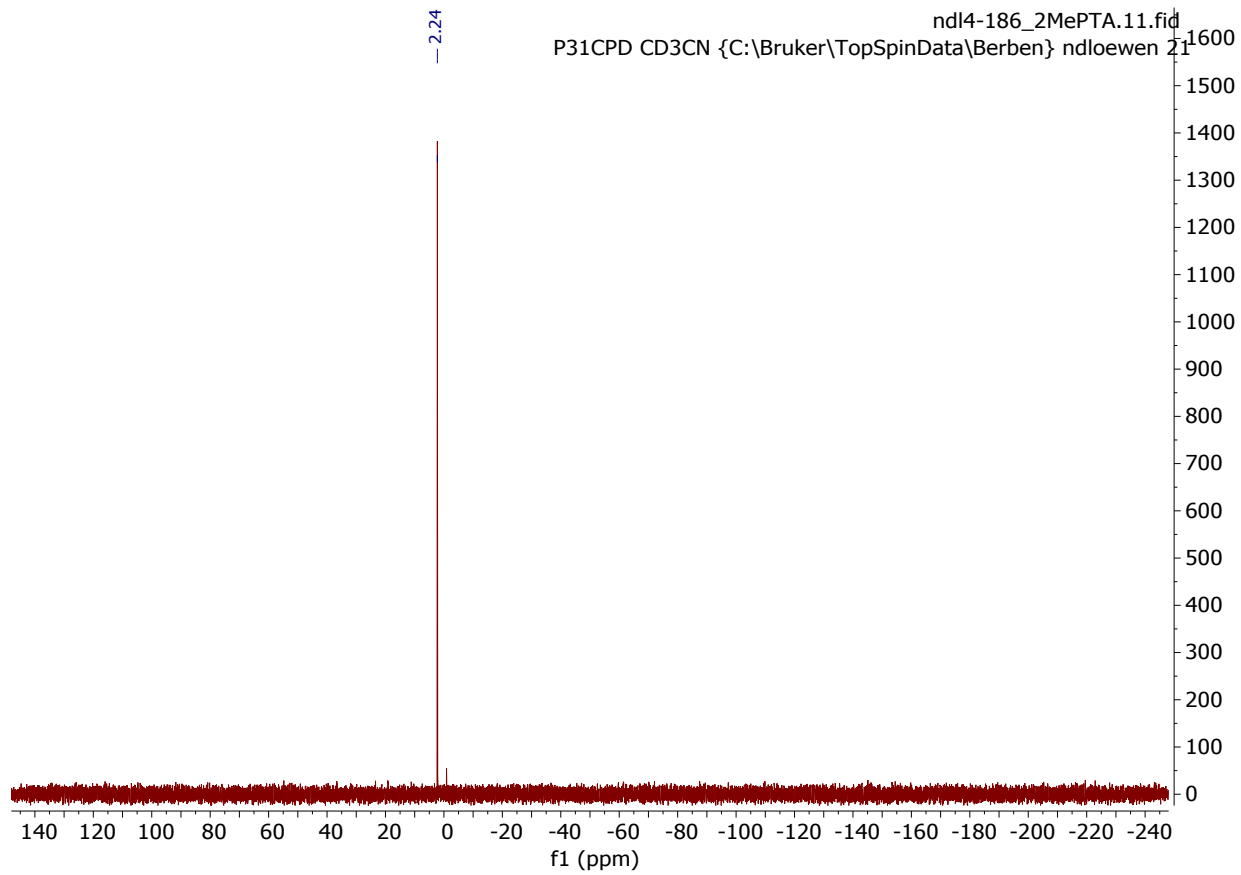

Figure S19. ${ }^{31} \mathrm{P}\left\{{ }^{1} \mathrm{H}\right\} \mathrm{NMR}\left(\mathrm{CD}_{3} \mathrm{CN}, 298 \mathrm{~K}, 400 \mathrm{MHz}\right)$ of 9-OTf. 


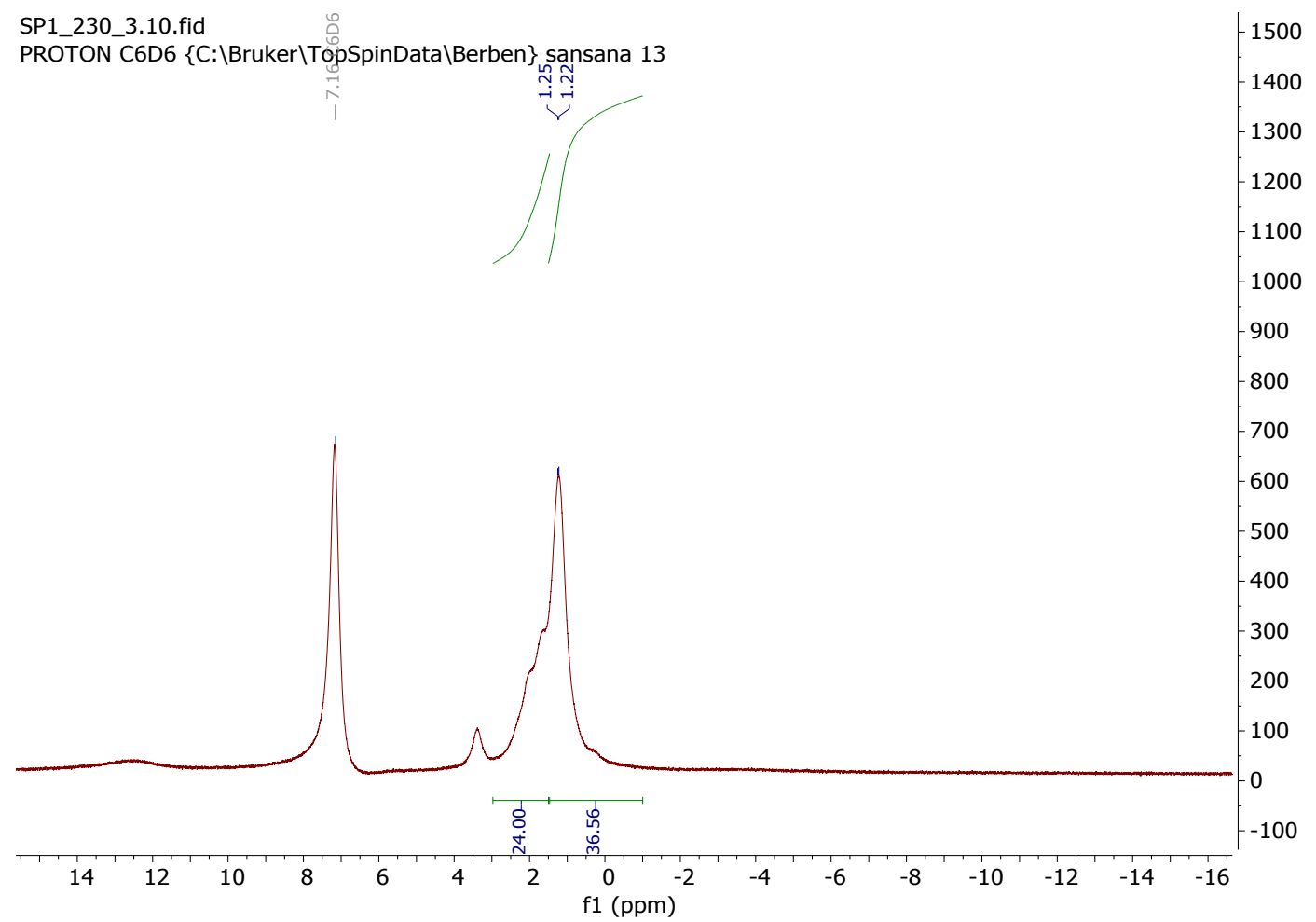

Figure S20. ${ }^{1} \mathrm{H}$ NMR $\left(\mathrm{C}_{6} \mathrm{D}_{6}, 298 \mathrm{~K}, 800 \mathrm{MHz}\right)$ of $\mathrm{K}-10$.

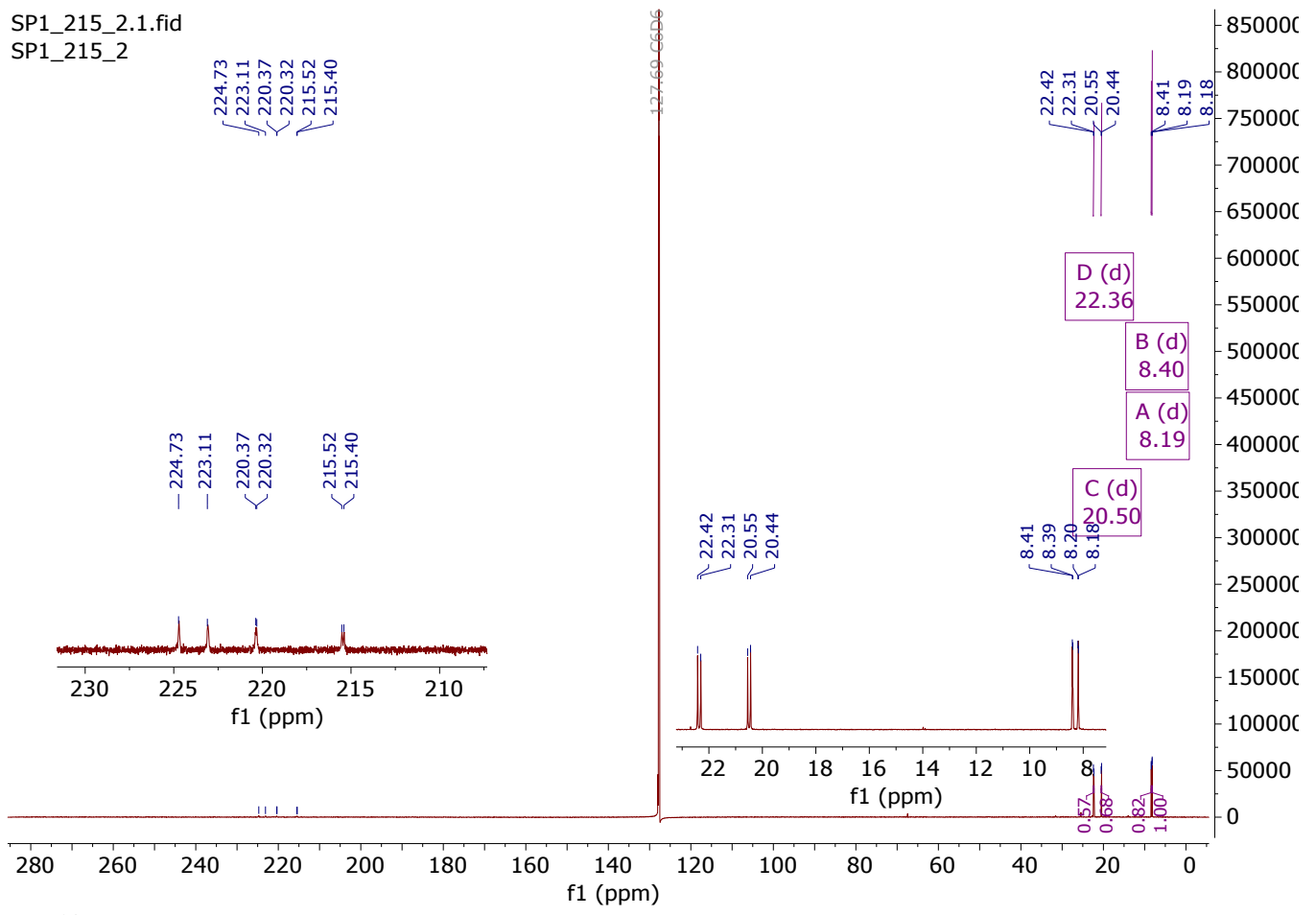

Figure 21. ${ }^{13} \mathrm{C}$ NMR $\left(\mathrm{C}_{6} \mathrm{D}_{6}, 298 \mathrm{~K}, 800 \mathrm{MHz}\right)$ of $\mathrm{K}-\mathbf{1 0}$. 


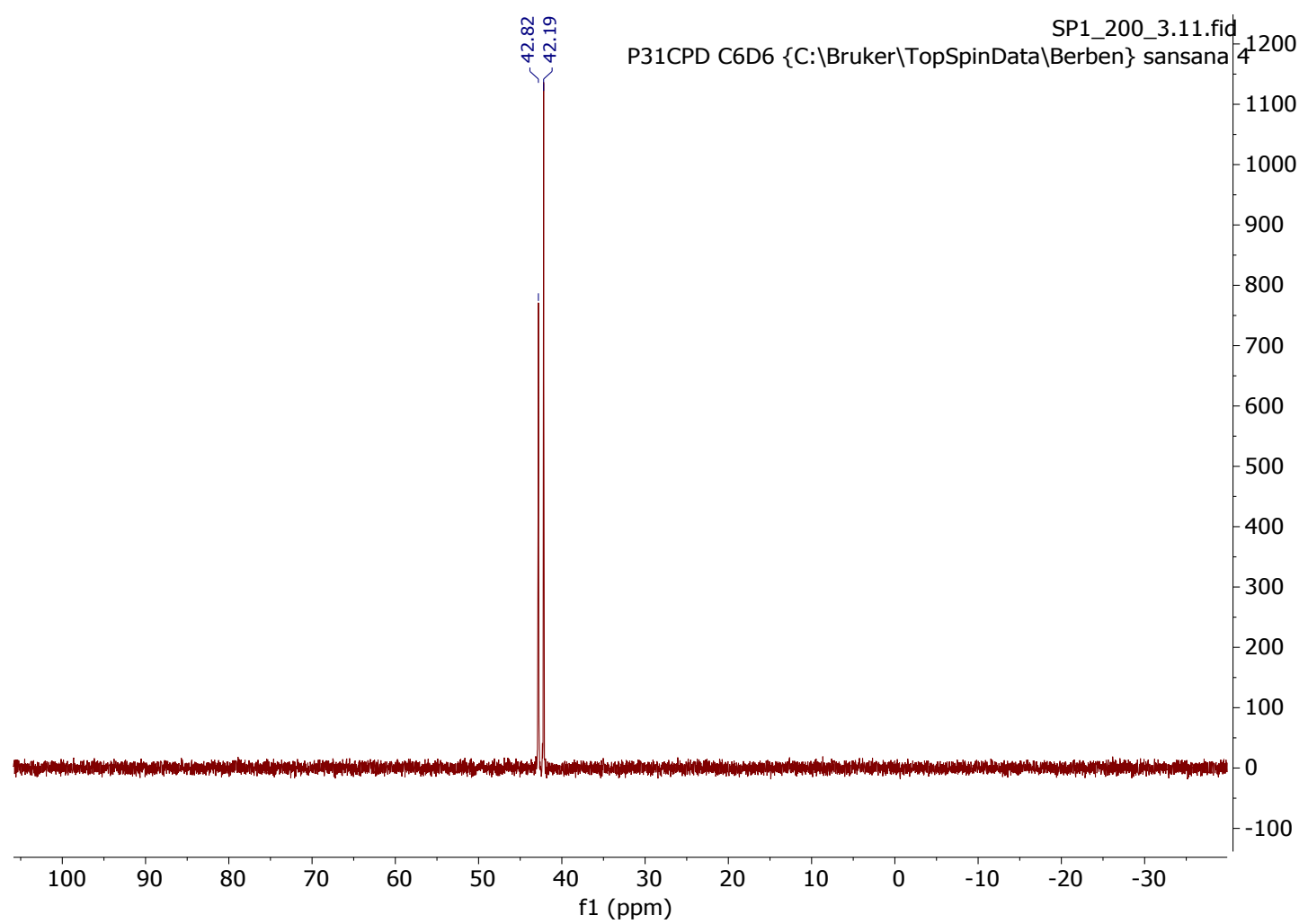

Figure S22. ${ }^{31} \mathrm{P}\left\{{ }^{1} \mathrm{H}\right\} \mathrm{NMR}\left(\mathrm{C}_{6} \mathrm{D}_{6}, 298 \mathrm{~K}, 800 \mathrm{MHz}\right)$ of $\mathrm{K}-\mathbf{1 0}$.

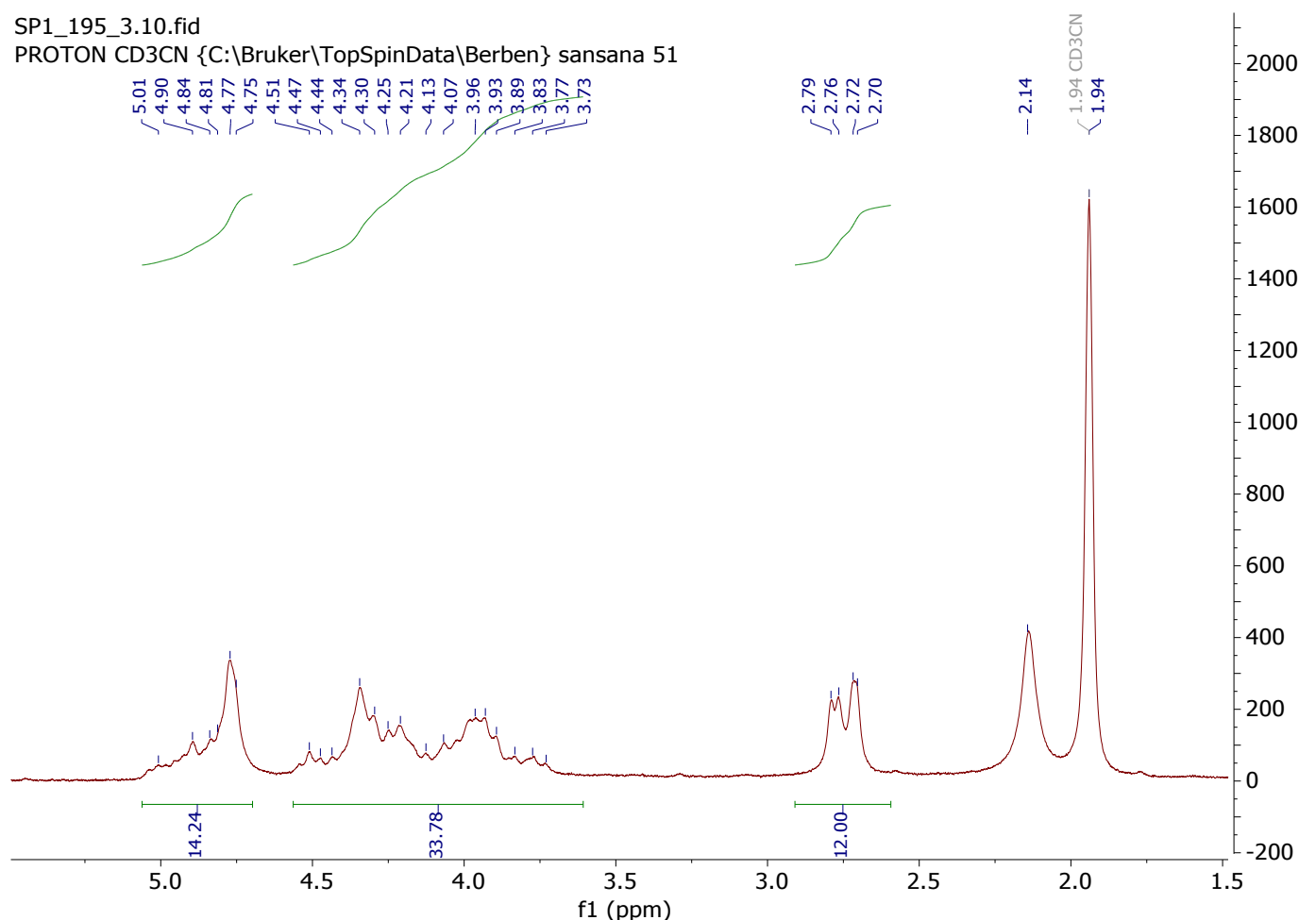

Figure S23. ${ }^{1} \mathrm{H} \mathrm{NMR}\left(\mathrm{CD}_{3} \mathrm{CN}, 298 \mathrm{~K}, 400 \mathrm{MHz}\right)$ of 11-OTf 3 . 


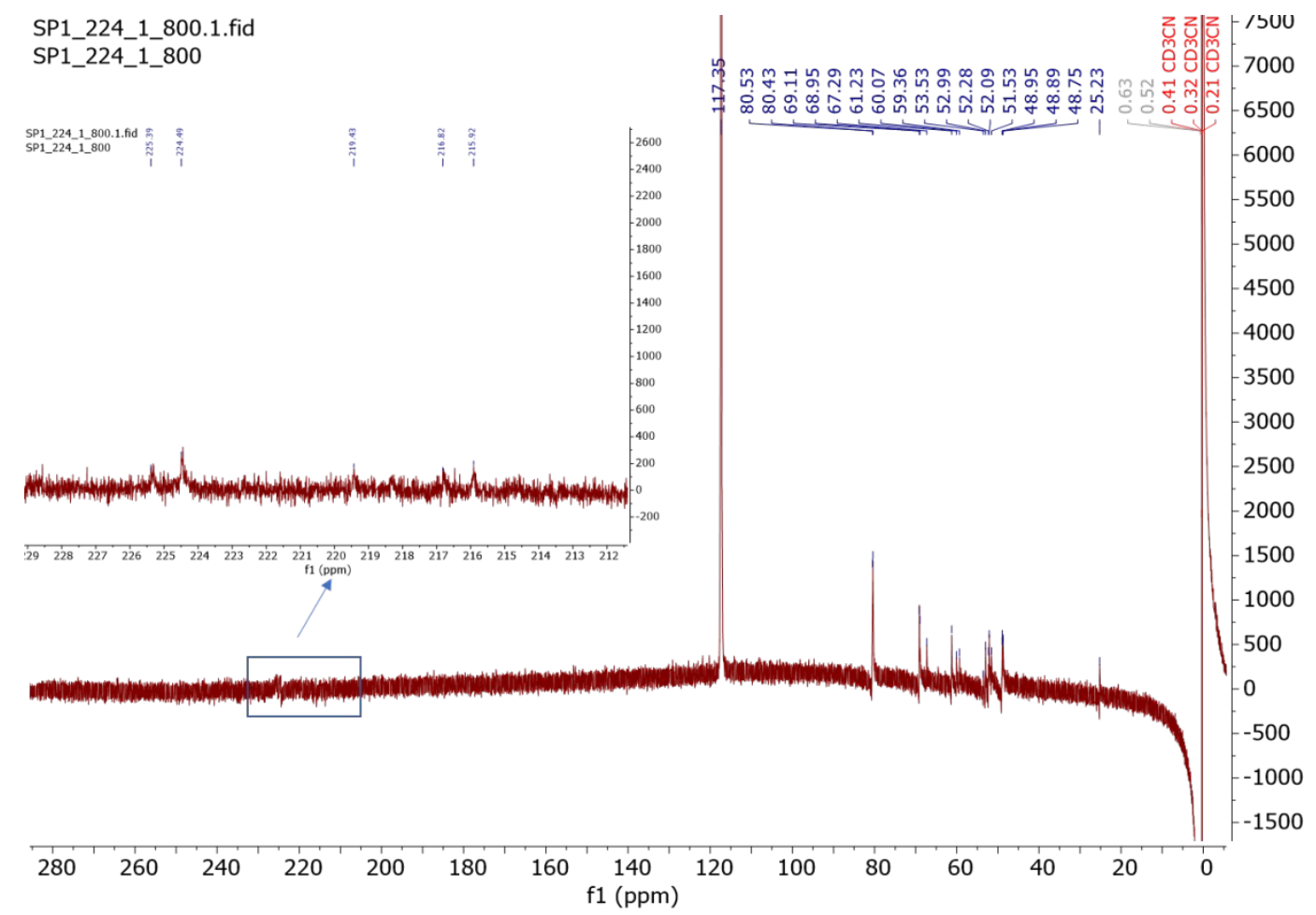

Figure S24. ${ }^{13} \mathrm{C} \mathrm{NMR}\left(\mathrm{CD}_{3} \mathrm{CN}, 298 \mathrm{~K}, 800 \mathrm{MHz}\right)$ of 11-OTf 3 .

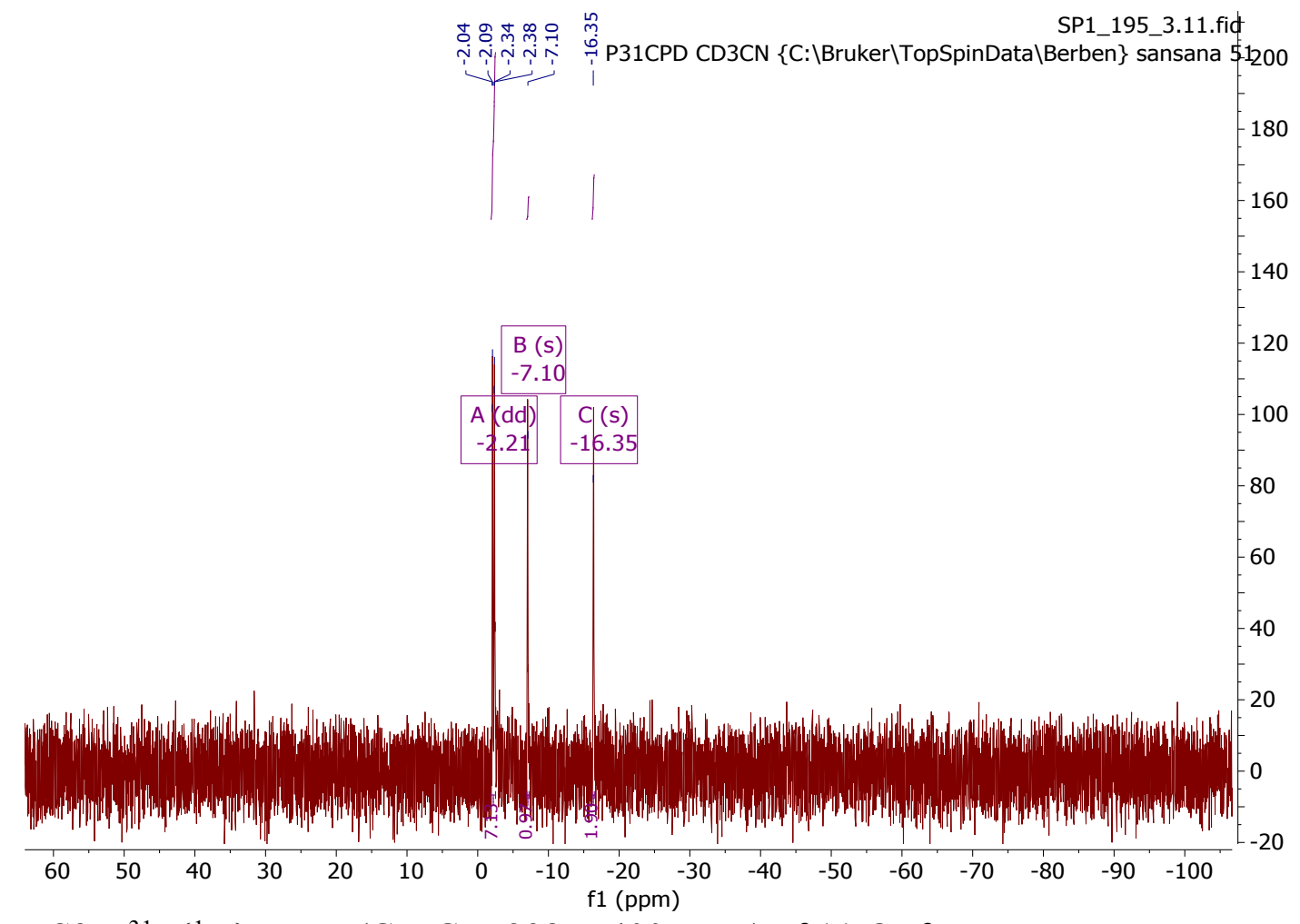

Figure S25. ${ }^{31} \mathrm{P}\left\{{ }^{1} \mathrm{H}\right\} \mathrm{NMR}\left(\mathrm{CD}_{3} \mathrm{CN}, 298 \mathrm{~K}, 400 \mathrm{MHz}\right)$ of 11-OTf 3 . 


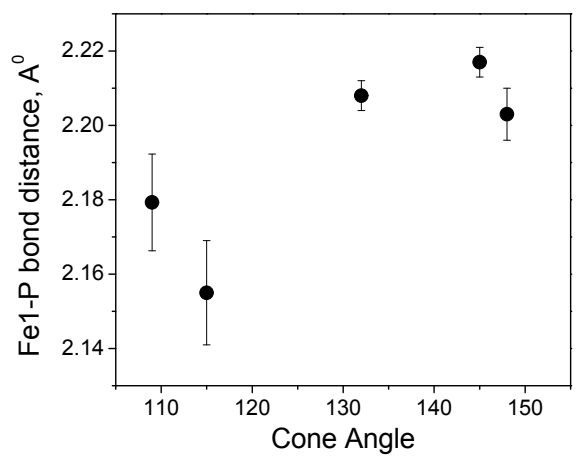

Figure S26. Plot of Fe1-P bond distances against cone angle of phosphines (from smallest to largest: PTA, MePTA, $\mathrm{PEt}_{3}, \mathrm{PPh}_{3}$ and $\mathrm{PPh}_{2} \mathrm{Py}$ ).

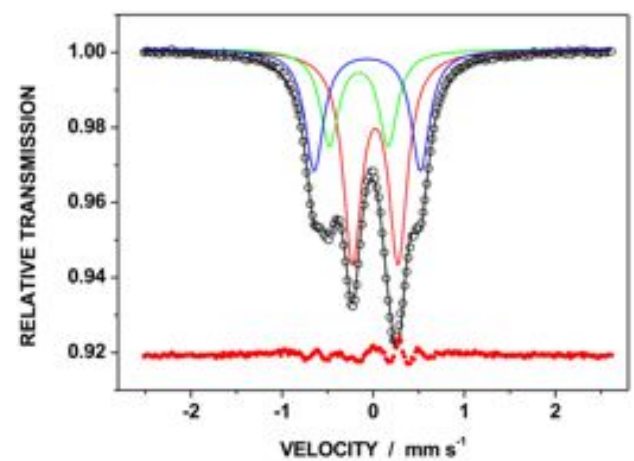

Figure S27. Zero field ${ }^{57} \mathrm{Fe}$ Mössbauer spectrum of $\mathrm{Et}_{4} \mathrm{~N}-6$. Green trace is Fe1, blue trace is Fe4, red trace is $\mathrm{Fe} 2$ and $\mathrm{Fe} 3$. The bottom trace (red) reflects the difference between the calculated and observed resonances.

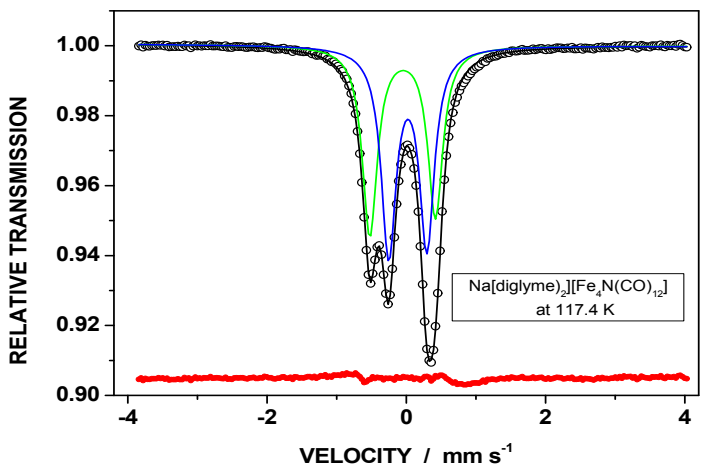

Figure S28. Zero field ${ }^{57} \mathrm{Fe}$ Mössbauer spectrum of Na-1. Blue trace is Fel and Fe4, green trace is $\mathrm{Fe} 2$ and $\mathrm{Fe} 3$. The bottom (red) trace is the difference between the calculated and experimental parameters and reflects the absence of impurity resonances. 


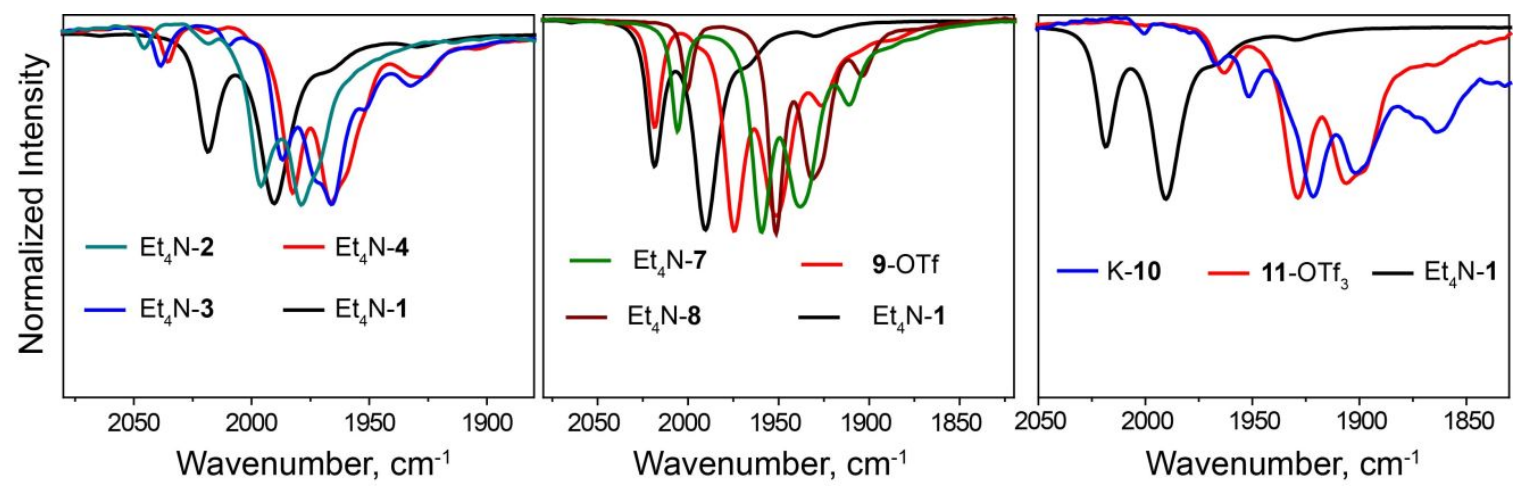

Figure S29. Infrared spectra of all phosphine substitued clusters (Left: one phosphine; Middle: two phosphine; Right: four phosphine substituted clusters) measured in acetonitrile solvent under $\mathrm{N}_{2}$ atmosphere.

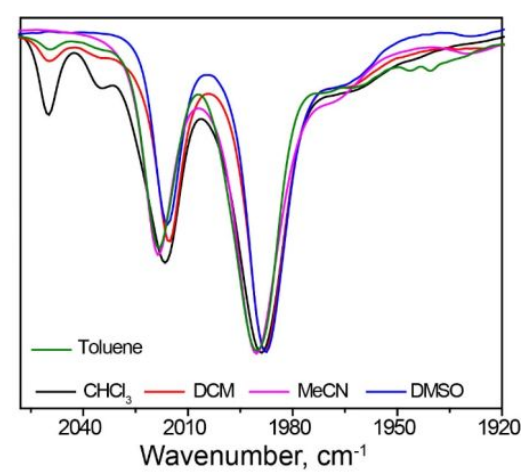

Figure S30. Normalized IR spectra of $0.01 \mathrm{mM} \mathrm{Et}_{4} \mathrm{~N}-1$ in different anhydrous solvent under $\mathrm{N}_{2}$ atmosphere.

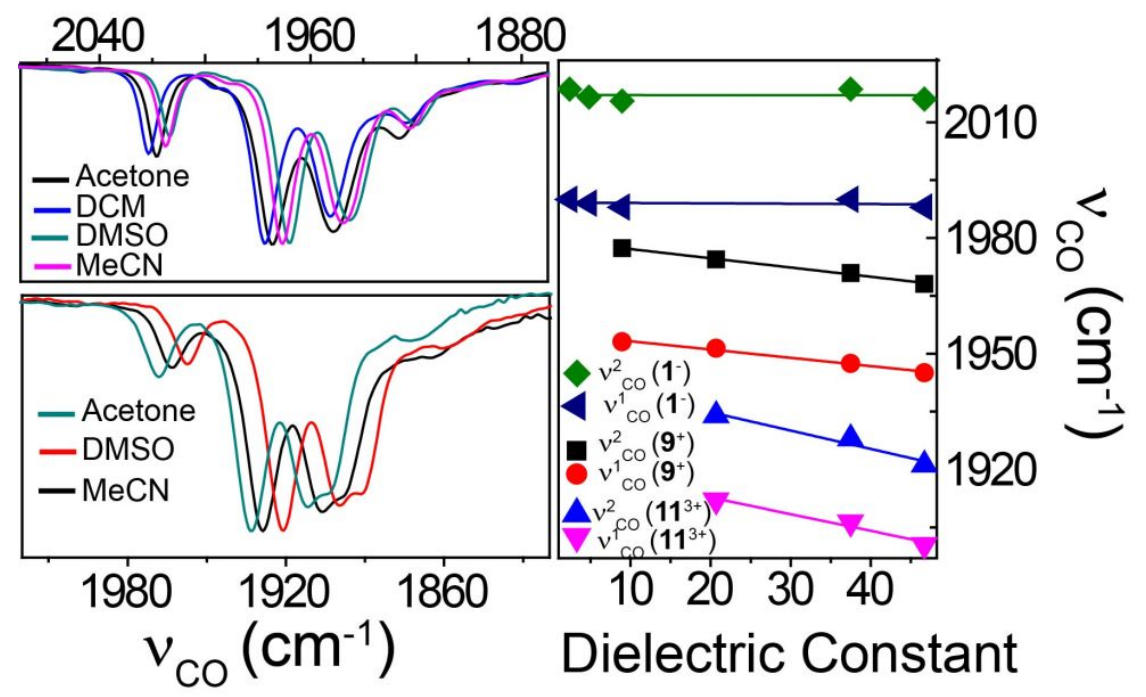

Figure S31: Normalized IR spectra of $0.01 \mathrm{mM} \mathrm{9}^{+}$(top left), $\mathbf{1 1}^{3+}$ (bottom left). (Right) Plot of $v_{\mathrm{CO}}$ bands for $\mathbf{1}^{-}, \mathbf{9}^{+}$and $\mathbf{1 1}^{3+}$ as a function of solvent dielectric constant $(\varepsilon):^{12} \varepsilon$ (Toluene) $2.38, \varepsilon$ (Chloroform) 4.81 ( $\varepsilon$ (Acetone) 20.7, $\varepsilon$ (DCM) 8.93, $\varepsilon(\mathrm{MeCN}) 37.5$ and $\varepsilon$ (DMSO) 46.7. 


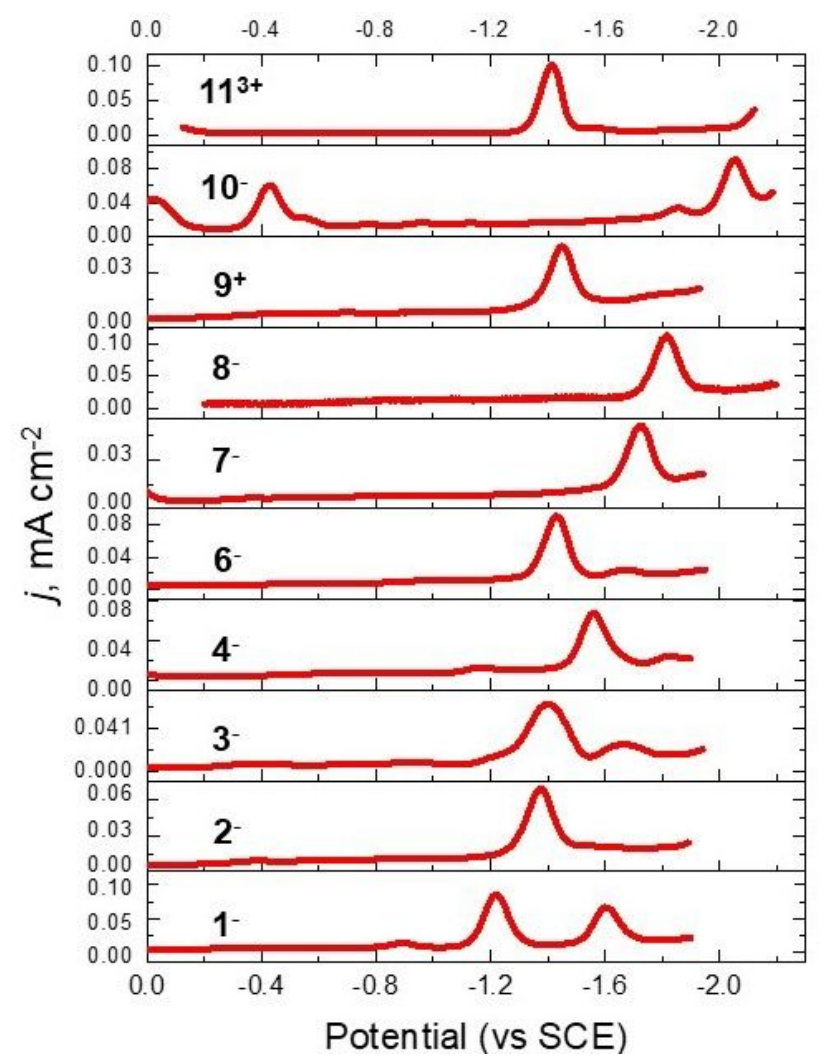

Figure S32. DPVs of $0.2 \mathrm{mM} \mathrm{1}^{-}-\mathbf{1 1}^{3+}$ collected in $0.1 \mathrm{M} \mathrm{Bu}_{4} \mathrm{NBF} / \mathrm{MeCN}$ under 1 atm $\mathrm{N}_{2}$. This is the same data shown in Figure 3 of the main text. Ferrocene was used as an external standard to calibrate potential vs SCE in MeCN. For $10^{-}$the peak at $-0.43 \mathrm{~V}$ is the $\mathbf{1 0}^{0 /-}$ couple, and for all other clusters the corresponding event lies just positive of $0 \mathrm{~V}$. The open circuit potential for 10is around $-0.8 \mathrm{~V}$ but we started the scan more anodic for consistency with the other traces. In some traces, including $\mathbf{1}^{-}$, the reduction event cathodic of the $\mathbf{1}^{-/ 2}$ - couple is the $\mathbf{1}^{2-13-}$ couple which has been previously reported by us, ${ }^{8}$ and others..$^{13}$ At about $-2.0 \mathrm{~V}$ the solvent/electrolyte reduction is observed a little and gives the rising baseline.

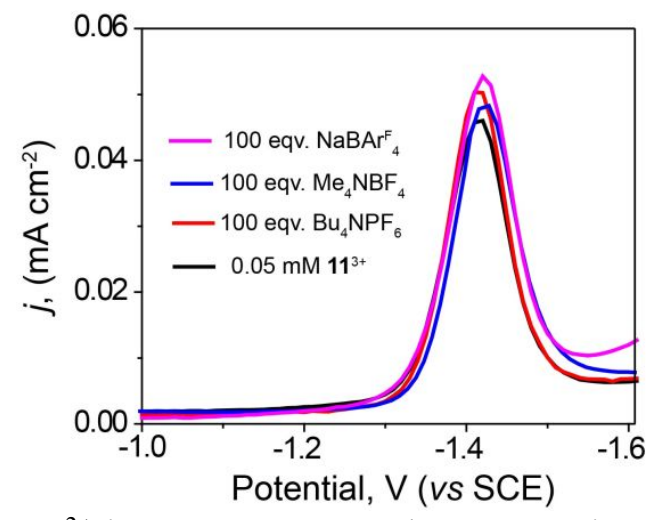

Figure 33. DPVs of $0.05 \mathrm{mM} \mathrm{11}{ }^{3+}$ in $0.1 \mathrm{M} \mathrm{Bu}_{4} \mathrm{NBF} / \mathrm{MeCN}$ under $1 \mathrm{~atm} \mathrm{~N}_{2}$. Different anion

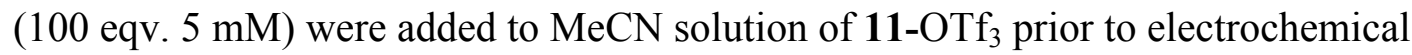
measurements. 


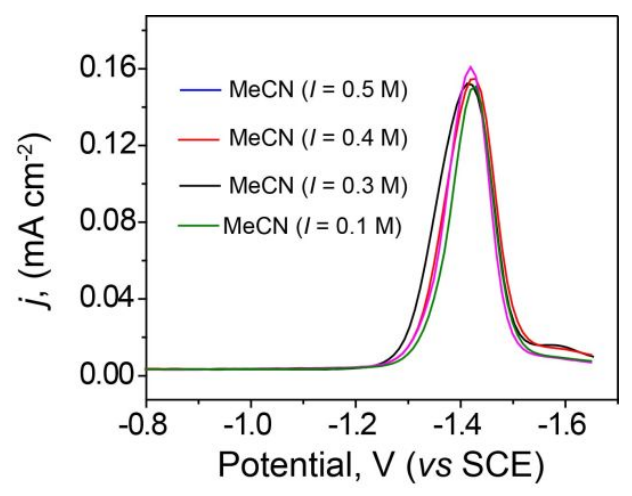

Figure S34. DPVs of $0.2 \mathrm{mM} \mathrm{11-OTf}_{3}$ in acetonitrile with different ionic strength $(I)$. The ionic strength of acetonitrile solution was maintained using different amount of tetrabutyl ammonium tetrafluoroborate salt $\left(\mathrm{Bu}_{4} \mathrm{NBF}_{4}\right)$ under $\mathrm{N}_{2}$ atmosphere.

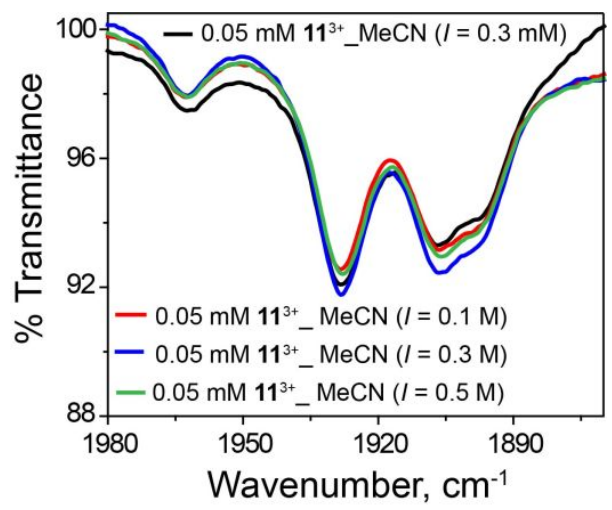

Figure S35. IR spectra of $0.05 \mathrm{mM} \mathrm{11-OTf} \mathrm{O}_{3}$ in acetonitrile with different ionic strength $(I)$. The ionic strength of acetonitrile solution was maintained using different amount of tetrabutyl ammonium tetrafluoroborate salt $\left(\mathrm{Bu}_{4} \mathrm{NBF}_{4}\right)$ under $\mathrm{N}_{2}$ atmosphere. Ionic strength of $0.05 \mathrm{mM}$ 11-OTf $f_{3}(I=0.3 \mathrm{mM})$ in acetonitrile calculated using $I=1 / 2 \Sigma\left(\mathrm{C}_{\mathrm{i}} \mathrm{Z}_{\mathrm{i}}^{2}\right)$ equation, $\mathrm{C}$ and $\mathrm{Z}$ stands for concentration and charge of respective ions.

\section{References}

${ }^{1}$ SMART Software Users Guide, Version 5.1, Bruker Analytical X-Ray Systems, Inc., Madison, WI, 1999.

2 Bruker (2019) APEX3 (Version 2019.1) and (2016) SAINT (Version 8.37a). Bruker AXS Inc., Madison, Wisconsin, USA.

${ }^{3}$ (a) Blessing, R. H. An Empirical Correction for Absorption Anisotropy. Acta Crystallogr., Sect. A: Found. Adv 1995, 51, 33-38; (b) Sheldrick, G.M., SADABS (2016) Version 2016/2, 'Siemens Area Detector Absorption Correction’ Universität Göttingen: Göttingen, Germany.

${ }^{4}$ (a) Sheldrick, G. M. (2014) SHELXT, Universität Göttingen: Göttingen, Germany. Structure determination program. Private communication; (b) Sheldrick, G. M. (2017). SHELXL2017/1. Universität Göttingen: Göttingen, Germany. 
${ }^{5}$ House, H. O.; Feng, E.; Peet, N. P. A Comparison of Various Tetraalkylammonium Salts as Supporting Electrolytes in Organic Electrochemical Reactions. J. Org. Chem. 1970, 36, 23712375.

${ }^{6}$ Dhar, D.; Yee, G. M.; Tolman, W. B. Effects of Charged Ligand Substituents on the Properties of the Formally Copper(III)-Hydroxide ([CuOH](2+)) Unit. Inorg. Chem. 2018, 57, 9794-9806.

${ }^{7}$ Mani, T.; Grills, D. C.; Miller, J. R. Vibrational Stark Effects to Identify Ion Pairing and Determine Reduction Potentials in Electrolyte-Free Environments. J. Am. Chem. Soc. 2015, 137, $1136-1140$.

8 (a) Rail, M. D.; Berben, L. A. Directing the Reactivity of $\left[\mathrm{HFe}_{4} \mathrm{~N}(\mathrm{CO})_{12}\right]^{-}$Toward $\mathrm{H}^{+}$or $\mathrm{CO}_{2}$ Reduction by Understanding the Electrocatalytic Mechanism. J. Am. Chem. Soc. 2011, 133, 18577-18579; (b) Nguyen, A. D.; Rail, M. D.; Shanmugam, M.; Fettinger, J. C.; Berben, L. A. Electrocatalytic Hydrogen Evolution from Water by a Series of Iron Carbonyl Clusters. Inorg. Chem. 2013, 52, 12847-12854.

${ }^{9}$ Loewen, N. D.; Berben, L. A. Secondary Coordination Sphere Design to Modify Transport of Protons and $\mathrm{CO}_{2}$. Inorg. Chem. 2019, 58, 16849-16857.

${ }^{10}$ Loewen, N. D.; Thompson, E. J.; Kagain, M.; Banales, C. L.; Myers, T. W.; Fettinger, J. C.; Berben, L. A. A pendant proton shuttle on $\left[\mathrm{Fe}_{4} \mathrm{~N}(\mathrm{CO})_{12}\right]^{-}$alters product selectivity in formate vs. $\mathrm{H}_{2}$ production via the hydride $\left[\mathrm{H}-\mathrm{Fe}_{4} \mathrm{~N}(\mathrm{CO})_{12}\right]$. Chem. Sci. 2016, 7, 2728-2735.

${ }^{11}$ Daigle, D. J., Decuir, T. J., Robertson, J. B. and Darensbourg, D. J. 1,3,5-Triaz-7-Phosphatricyclo[3.3.1.1, ${ }^{3,7}$ Decane and Derivatives. In Inorg. Synth., M. Y. Darensbourg (Ed.) (2007).

${ }^{12}$ Haynes, W. M.; Lide, D. R.; and Bruno, T. J. CRC handbook of chemistry and physics: a ready-reference book of chemical and physical data. 2016-2017, 97th Edition / Boca Raton, Florida: CRC Press (2016).

${ }^{13}$ Zanello, P.; Laschi, F.; Cinquantini, A.; Della Pergola, R.; Garlaschelli, L.; Cucco, M.; Demartin, F.; Spalding, T. R. The Redox Behaviour of the Cluster Anion $\left[\mathrm{Fe}_{4} \mathrm{~N}(\mathrm{CO})_{12}\right]^{-}$. Electron Transfer Chain Catalytic Substitution Reactions. Crystal Structure of $\left.\left[\mathrm{Fe}_{4} \mathrm{~N}(\mathrm{CO})_{11} \mathrm{PPh}_{3}\right)\right]^{-}$. Inorg. Chim. Acta 1994, 226, 1-8. 